ARTICLE

https://doi.org/10.1038/s41467-019-12656-x

\title{
A kinase-independent role for CDK8 in BCR-ABL1+ leukemia
}

Ingeborg Menzl (1) 1, Tinghu Zhang', Angelika Berger-Becvar ${ }^{1}$, Reinhard Grausenburger', Gerwin Heller 1,3,4, Michaela Prchal-Murphy ${ }^{1}$, Leo Edlinger ${ }^{1}$, Vanessa M. Knab¹, Iris Z. Uras', Eva Grundschober ${ }^{1}$, Karin Bauer ${ }^{5}$, Mareike Roth ${ }^{6}$, Anna Skucha7,8, Yao Liu², John M. Hatcher ${ }^{2}$, Yanke Liang ${ }^{2}$, Nicholas P. Kwiatkowski², Daniela Fux ${ }^{1}$, Andrea Hoelbl-Kovacic ${ }^{1}$, Stefan Kubicek (10) ${ }^{8}$, Junia V. Melo ${ }^{9,10}$, Peter Valent ${ }^{5}$,

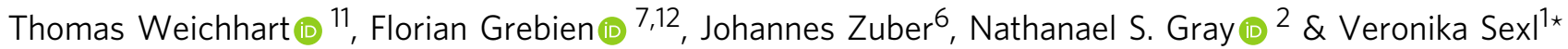

Cyclin-dependent kinases (CDKs) are frequently deregulated in cancer and represent promising drug targets. We provide evidence that CDK8 has a key role in B-ALL. Loss of CDK8 in leukemia mouse models significantly enhances disease latency and prevents disease maintenance. Loss of CDK8 is associated with pronounced transcriptional changes, whereas inhibiting CDK8 kinase activity has minimal effects. Gene set enrichment analysis suggests that the mTOR signaling pathway is deregulated in CDK8-deficient cells and, accordingly, these cells are highly sensitive to mTOR inhibitors. Analysis of large cohorts of human $A L L$ and AML patients reveals a significant correlation between the level of CDK8 and of mTOR pathway members. We have synthesized a small molecule YKL-06-101 that combines mTOR inhibition and degradation of CDK8, and induces cell death in human leukemic cells. We propose that simultaneous CDK8 degradation and mTOR inhibition might represent a potential therapeutic strategy for the treatment of ALL patients.

\footnotetext{
${ }^{1}$ Institute of Pharmacology and Toxicology, University of Veterinary Medicine, Vienna, Austria. ${ }^{2}$ Department of Cancer Biology, Department of Biological Chemistry and Molecular Pharmacology, Dana-Farber Cancer Institute, Harvard Medical School, Boston, Massachusetts, USA. ${ }^{3}$ Department of Medicine I, Medical University of Vienna, Vienna, Austria. ${ }^{4}$ Comprehensive Cancer Center, Vienna, Austria. ${ }^{5}$ Division of Hematology and Hemostaseology, Department of Internal Medicine I, Ludwig Boltzmann Institute for Hematology and Oncology, Medical University of Vienna, Vienna, Austria. ${ }^{6}$ Research Institute of Molecular Pathology, Campus-Vienna-Biocenter 1, Vienna, Austria. ${ }^{7}$ Ludwig Boltzmann Institute for Cancer Research, Vienna, Austria. ${ }^{8}$ Research Center for Molecular Medicine of the Austrian Academy of Sciences, Vienna, Austria. ${ }^{9}$ Faculty of Health and Medical Sciences, University of Adelaide, Adelaide, South Australia 5005, Australia. ${ }^{10}$ Department of Hematology, Imperial College London, Kensington, London SW7 2AZ, UK. ${ }^{11}$ Center of Pathobiochemistry and Genetics, Institute of Medical Genetics, Medical University of Vienna, Vienna, Austria. ${ }^{12}$ Institute for Medical Biochemistry, University of Veterinary Medicine, Vienna, Austria. *email: veronika.sexl@vetmeduni.ac.at
} 
C yclin-dependent kinases (CDKs) are serine/threonine kinases that are regulated by binding to cyclins ${ }^{1}$. CDKs were initially shown to play important roles in cell cycle control. Over time, their broad and diverse roles in many biological processes were uncovered and CDKs are now considered as important players in transcription, metabolism, neuronal differentiation, hematopoiesis, and stem cell self-renewal [reviewed in ref. ${ }^{2}$ ]. CDKs comprise two major sub-groups; the first CDK family including CDK1, 2, 4, and 6 is predominantly involved in cell cycle control. The second group comprising CDKs 7 through 13 are modulators of transcriptional processes ${ }^{3}$. CDK6 unifies functions of both groups ${ }^{4-8}$. This dual function may underlie the great success of three independent CDK4/6 inhibitors (palbo-, ribo-, and abemaciclib), which were recently declared as therapeutic breakthrough by the $\mathrm{FDA}^{9}$. Besides CDK4/6, also CDK7 and CDK9 have drawn considerable attention as drug targets. Both CDK7 and CDK9 phosphorylate serine residues in the C-terminal tail of RNA Polymerase II. CDK9 regulates transcription of key genes in hematological cancers such as myeloid cell leukemia-1 (MCL-1), B-cell lymphoma extra-long (BCL- $\mathrm{x}_{\mathrm{L}}$ ), or X-linked inhibitor of apoptosis protein (XIAP) ${ }^{10}$. CDK7 is considered to drive super-enhancer-(SE)-associated gene expression in neuroblastoma ${ }^{11}$ and T-cell acute lymphoid leukemia (T$\mathrm{ALL})^{12}$. First-generation CDK inhibitors such as flavopiridol ${ }^{13}$ and dinaciclib ${ }^{14}$ are active in acute myeloid leukemia (AML) ${ }^{15}$ and chronic myeloid leukemia (CML), but clinical benefit is limited due to adverse effects ${ }^{16,17}$.

CDK8 was initially reported to exert transcriptional repressive functions as part of the mediator complex, a core component of the basal transcription machinery. CDK8 and its paralog CDK19 bind to the mediator complex in a mutually exclusive way but both rely on binding of cyclin $\mathrm{C}$ (CCNC) for kinase activity ${ }^{3}$. Single knockout of $C d k 8$ and $C C N C$ results in embryonic lethality at E2.5-3 due to preimplantation defects ${ }^{18}$, whereas conditional deletion of CDK8 in adult mice is surprisingly well tolerated ${ }^{19}$. Recent studies have shown that CDK8 can exert activating functions as a co-regulator of $\mathrm{p} 53^{20}$ or hypoxia-induced gene expression $^{21}$. STAT transcription factors are among the bestdescribed targets of CDK8 $8^{22,23}$. Phosphorylation of STAT1 ${ }^{\text {S727 }}$ enhances transcriptional activity and results in interferon (IFN)induced gene transcription ${ }^{24}$.

The role of CDK8 appears to be divergent and highly contextdependent. In colon cancer $^{25,26}$, melanoma ${ }^{27}$, prostate ${ }^{28}$, and breast cancer ${ }^{29}$, CDK8 accelerates proliferation and migration. In contrast, it acts as a tumor suppressor in endometrial ${ }^{30}$ and intestinal tumors ${ }^{19}$. In some AML cell lines, inhibition of CDK8 via steroidal alkaloid cortistatin A dramatically alters gene expression and blocks cell proliferation. These changes were due to the relief of CDK8-mediated repression of SE-driven transcription $^{31}$.

The BCR-ABL1 fusion protein drives the development of CML and a subset of ALL cases, which are considered a particular therapeutic challenge. Albeit tyrosine kinase inhibitors (TKIs) for the BCR-ABL1 oncoprotein are available, further therapeutic improvement is required ${ }^{32}$. Resistance mechanisms towards TKIs demand the development of therapeutic strategies ${ }^{33}$. Our findings identify CDK8 as a key mediator of BCR-ABL1-driven leukemia. The role of CDK8 goes beyond its kinase activity, suggesting the development of therapeutic strategies towards its kinaseindependent functions.

\section{Results}

CDK8 is essential for survival of BCR-ABL1p185+ leukemic cells. To investigate which CDKs are expressed in hematopoietic malignancies, we measured the levels of CDK6, CDK7, CDK8,
CDK9, and CDK19 in a panel of human leukemic cell lines by immunoblotting. Irrespective of the cells' origin, the levels of CDK6, CDK7, CDK8, CDK9, and CDK19 were dramatically increased in all cell lines compared with non-transformed human mononuclear lymphocytes (hMNL). CDK8 is part of the kinase submodule of the mediator complex, so we tested whether the other members of this complex are also upregulated and we found increased levels of MED12, MED13, and CCNC, which are part of the mediator kinase module (Fig. 1a). A comparable situation was found in murine leukemia cell lines transformed by the $\mathrm{v}-\mathrm{ABL} \mathrm{p}^{\mathrm{1} 60+}$ or BCR-ABL1p185+ oncogenes (Fig. 1b).

We tested which of the CDKs support viability and proliferation of mouse BCR-ABL1 ${ }^{\text {p185+ }}$ B-ALL cells by using an inducible Tet-On RNAi system, in which short hairpin RNA (shRNA) expression is coupled to a dsRed reporter gene. DsRed ${ }^{+}$cells were detectable 2 days after doxycycline treatment and followed over time. Although knockdown of CDK6, 7, or 9 did not induce strong anti-proliferative responses, we found a pronounced reduction in the frequency of $\mathrm{dsRed}^{+}$cells upon knockdown of CDK8 comparable to MYC knockdown, a positive control (Fig. 1c). This prompted us to include CDK19, which has functional homology to CDK8, and the CDK8-binding partners CCNC and MED12, which regulate CDK8's kinase activity. Neither of these knockdown approaches fully recapitulated the effects of loss of CDK8, with the exception of MED12 (Fig. 1c). Efficiencies of CDK6, CDK7, CDK9, CDK19, MED12, and CCNC knockdown were verified by immunoblotting (Fig. $1 \mathrm{~d}$ and Supplementary Fig. 1a). Downregulation of CDK8, MED12, or c-MYC was less pronounced, indicating that loss of either protein is incompatible with survival. Growth curves supported these observations (Fig. 1e).

Stable shRNA-mediated knockdown for CDK9, CDK19, MED12, MED13, or CCNC in four individually derived murine BCR-ABL1p185+ cell lines gave similar effects to the inducible knockdowns. However, we failed to obtain lines deficient for CDK8 or CDK7 (Supplementary Fig. 1b). We have no explanation for the different effects of CDK7 deletion in shortand long-term knockdown experiments. Our failure to generate CDK8-deficient lines shows that loss of CDK8 is incompatible with survival of BCR-ABL1p185+ cells.

Steady-state hematopoiesis is not affected by loss of CDK8. Efficient drug treatment requires a sufficiently large therapeutic window, which allows killing of tumor cells while sparing normal tissue. We therefore investigated the consequences of $C d k 8$ deletion on normal, non-leukemic hematopoiesis using $C d k 8^{\Delta / \Delta}$ Vav-Cre mice. Bone marrow (BM) was isolated from 6-week-old mice. Efficient deletion of CDK8 was verified by immunoblotting (Fig. 2a). Overall, the loss of CDK8 was well tolerated, as white blood cell counts (WBCs), red blood cell counts (RBCs) and numbers of platelets were comparable to those of control mice (Fig. 2b). Detailed flow cytometric analyses revealed no significant differences in the frequencies of hematopoietic cells at various stages of differentiation, indicating that hematopoiesis remained largely unaffected under steady-state conditions (Fig. 2c). Importantly, percentages of LSK cells and stem cell sub-fractions were comparable (Fig. 2d-e). As BCRABL1 $1^{\text {p185+ }}$ cells are of B-lymphoid origin, we closely investigated the consequences of CDK8 deficiency on early B-cell development. B-cell developmental stages can be distinguished by differential cell-surface expression of B220, CD43, CD19, BP-1, IgM, and $\operatorname{IgD}^{34,35}$. Frequencies of individual B-cell fractions were unaltered in BMs of $C d k 8^{\Delta / \Delta}$ Vav-Cre mice (Fig. 2f). Poly(I:C) treatment in $C d k 8^{\Delta / \Delta} \mathrm{Mx} 1 \mathrm{Cre}$ mice was used to challenge stressinduced hematopoiesis as it induces a type I IFN-triggered 
a

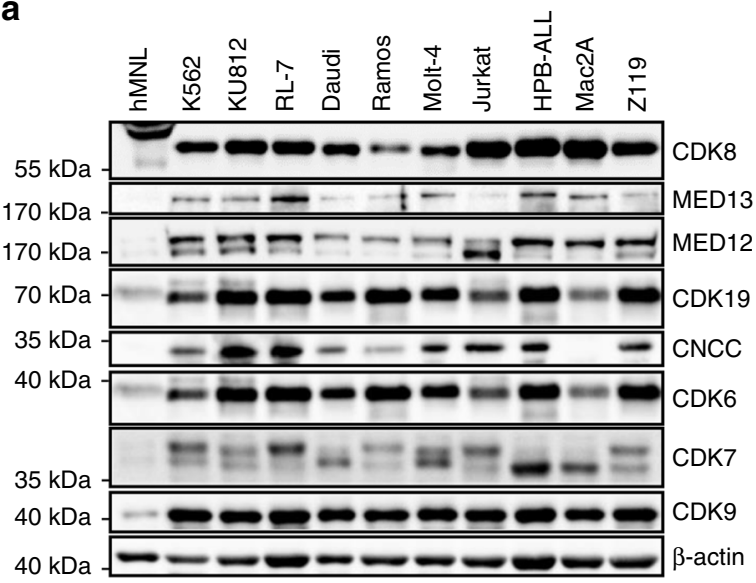

b

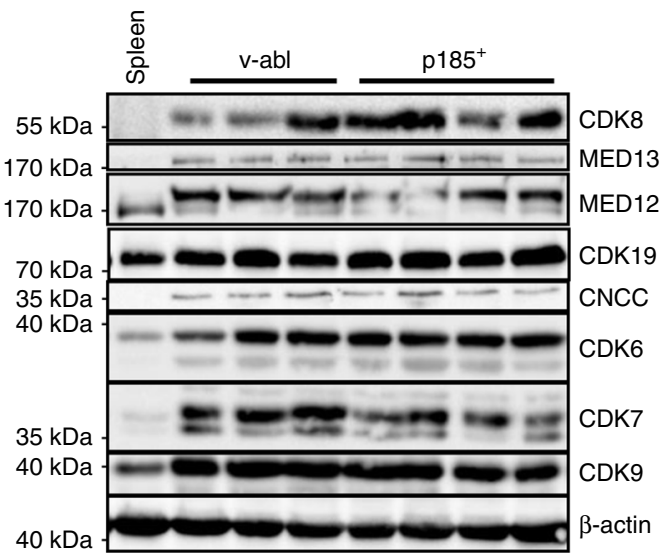

C

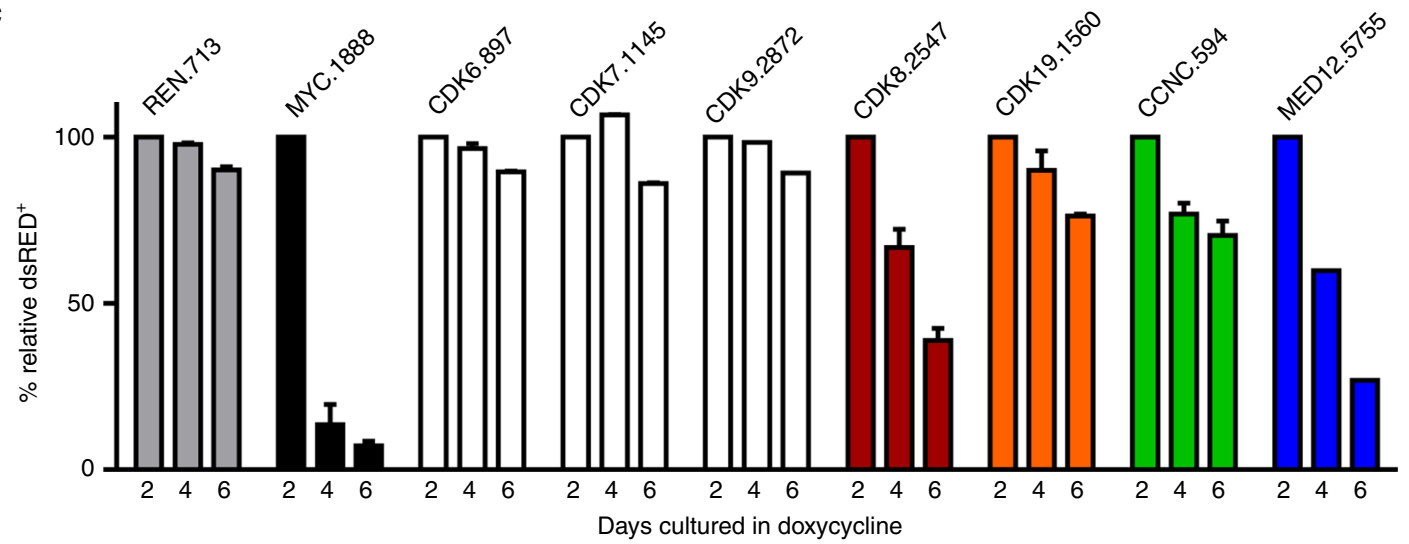

d
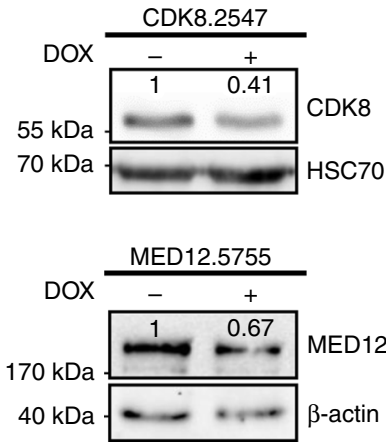

e

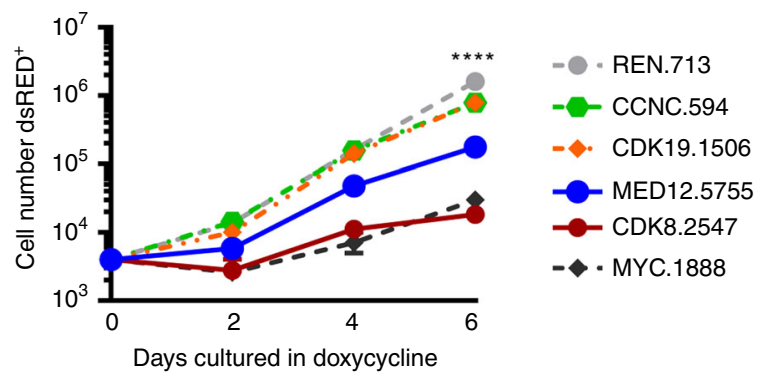

Fig. 1 CDK8 is essential for survival of BCR-ABL1185+ leukemic cells. Immunoblotting: levels of CDK6, CDK7, CDK8, CDK9, CDK19, CCNC, MED12, and MED13 in leukemic human (a) and murine (b) cell lines. Levels of $\beta$-actin served as loading control. c Induction of shRNA-mediated knockdowns by doxycycline. Percentages of dsRED+ BCR-ABL1185+ leukemic cells transduced with TRE3G-dsRED-shRNA-puro (Tet-On) targeting CDK6, CDK7, CDK8, CDK9, CDK19, CNCC, or MED12. Numbers indicate the starting point of shRNA sequence. Data represent frequencies of dsRed ${ }^{+}$BCR-ABL1p185+ cells over time, normalized to the percentages of dsRED + cells after 2 days of doxycycline (DOX) administration. shRNAs directed against Renilla (REN) or MYC served as negative and positive controls. One representative experiment performed in duplicates out of three with similar outcome is shown. d Verification of shRNA-mediated knockdown of CDK8 and MED12 by immunoblotting (day 2 after doxycycline administration). $\beta$-Actin and HSC70 served as a loading control. Numbers refer to densitometric analysis of the blotted protein in reference to loading control levels. e Growth curves of shRNA-expressing (dsRed ${ }^{+}$) Tet-On BCR-ABL1p185+ cells. One representative experiment performed in triplicates out of three with similar outcome is shown. Levels of significance were calculated using two-way ANOVA followed by Dunn's test; data represents means \pm SD ( ${ }^{\star \star \star \star} p<0.0001$ ). Source data are provided as a Source Data file

ubiquitous deletion of Cdk8. Poly(I:C) treatment in $C d k 8^{f l}$ ${ }^{l}$ Mx1Cre mice reflected the observations in $C d k 8^{\Delta / \Delta} \mathrm{Vav}$-Cre mice; we did not detect significant changes in the frequencies of hematopoietic cell subsets in the BM or in the blood cells upon Cdk8 deletion (Supplementary Fig. 2a-f). To explore the functionality of CDK8-deficient stem cells, we set up a competitive transplant experiment. We mixed BM cells from $C d k 8^{\Delta / \Delta} \mathrm{Vav}$-Cre $L y 5.2^{+}$or $C d k 8^{f l f l} L y 5.2^{+}$mice with $C D K 8^{+/+}$Ly5.1 $1^{+}$BM cells in a 1:1 ratio and injected them intravenously (i.v.) into lethally irradiated $L y 5.1 / 2^{+}$mice (Fig. $2 \mathrm{~g}$ ). Ten weeks after transplantation, no differences in the repopulation capacity of stem cells of different genotypes were observed (Fig. 2h, i). The absence of 


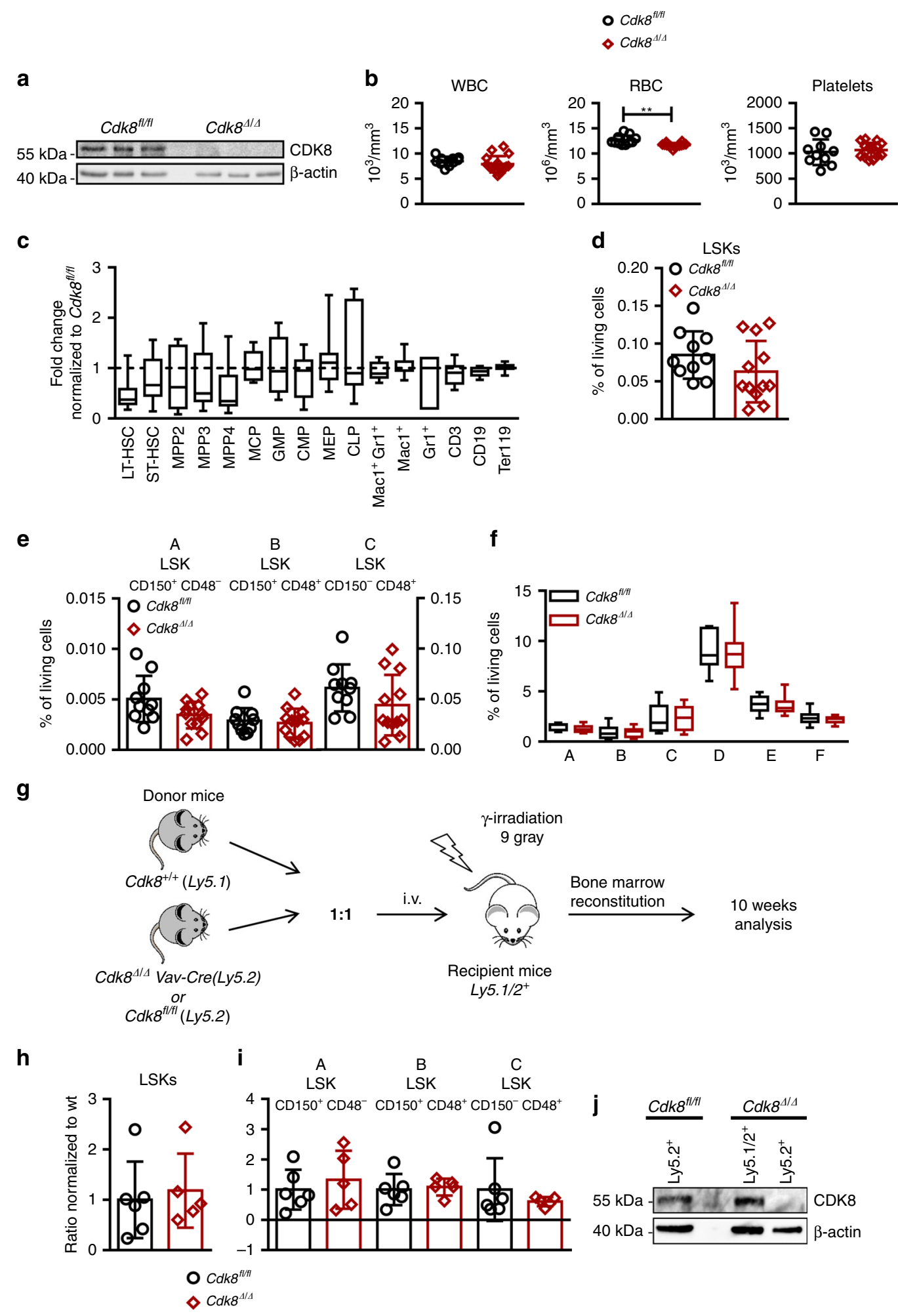

CDK8 was confirmed in sorted BM-derived Cdk8 $8^{\Delta / \Delta}$ Vav-Cre Ly5.2 $2^{+}$cells (Fig. $2 \mathrm{j}$ ). In summary, conditional and inducible loss of CDK8 in the hematopoietic system is well tolerated, underlining the potential of CDK8 as a therapeutic target.

CDK8 is not required for initial BCR-ABL1P185+ transformation. To test the consequences of $C d k 8$ deficiency for B-lymphoid transformation, we infected $C d k 8^{\Delta / \Delta}$ Vav-Cre-BM cells with a retrovirus encoding pMSCV-Bcr-Abl1p185-IRESeGFP or Ab-MuLV (encoding v-ABL ${ }^{160+}$ ). Infected cells were subsequently plated in growth factor-free methylcellulose. Irrespective of the genotype, comparable numbers of B-lymphoid colonies grew out (Fig. 3a and Supplementary Fig. 3a). Deficiency of CDK8 did not affect the levels of CDK19, MED12, MED13, or CNCC (Fig. 3b and Supplementary Fig. 3b). Transformation by $\mathrm{BCR}-\mathrm{ABL} 1^{\mathrm{p} 185+}$ or $\mathrm{v}-\mathrm{ABL} \mathrm{p}^{\mathrm{160}}+$ resulted in the outgrowth of 
Fig. 2 Steady-state hematopoiesis is not affected by loss of CDK8 (Cdk8 $8^{\Delta / \Delta V a v-C r e)}$. a Efficiency of CDK8 deletion in 6-week-old Cdk8 $8^{\Delta / \Delta}$ Vav-Cre mice. Immunoblotting of BM cells from $C d k 8^{f l / f l}$ and $C d k 8^{\Delta / \Delta} \operatorname{Vav}$-Cre mice $(n=3$ per genotype). $\mathbf{b}$ Analysis of white blood cell count (WBC), red blood cell count (RBC), and platelet count of $C d k 8^{f l / f l}(n=10)$ and $C d k 8^{\Delta / \Delta}$ Vav-Cre mice $(n=16)$ are depicted. c Relative fold change of BM composition of $C d k 8^{\Delta / \Delta} \mathrm{Vav}-\mathrm{Cre}$ mice $(n=10)$ normalized to mean of $C d k 8^{f / f f l}(n=10)$. Center value represents median, the box 25th to 75th percentiles, and whiskers min to max. d Bar diagram of $\mathrm{Lin}^{-} \mathrm{Sca}-1^{+} \mathrm{c}^{-k i t}{ }^{+}(\mathrm{LSK})$ frequencies in BMs of $\mathrm{Cdk} 8^{\mathrm{fl} / \mathrm{fl}}(n=10)$ and $\mathrm{Cdk} 8^{\Delta / \Delta} \mathrm{Vav}-\mathrm{Cre}(n=12)$ mice. e Frequencies of LSK subpopulations (fraction $A, B$, and $C ; C d k 8^{f l / f l} n=10, C d k 8^{\Delta / \Delta}$ Vav-Cre $n=12$ ). f Frequencies of individual populations during early B-cell development according to Hardy nomenclature in pre-pro-B (B220+/CD43hi/CD19-/BP-1-; fraction A), early pro-B (B220+/CD43hi/CD19+/BP-1-; fraction B), late pro-B

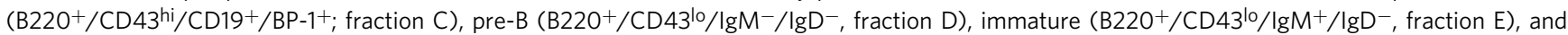

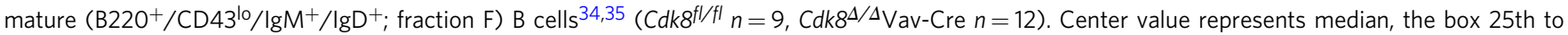
75th percentiles and whiskers min to max. $\mathbf{g}$ Experimental setup of competition transplants data shown in $\mathbf{h}$, i. $\mathbf{h}$ Bar graph displays LSK+ cells analyzed for Ly5.1+/Ly5.2+ composition in total BM. i Contributions of Ly5.1+ and Ly5.2+ cells in LSK subpopulations (fraction A, B, and C; Cdk8fl/fl $n=6$ and Cdk8 ${ }^{\Delta /}$ ${ }^{\Delta}$ Vav-Cre $\left.n=5\right)$. j Immunoblot of sorted BM cells after competitive transplant. Detection of CDK8 in Cdk8fl/fl Ly5.2, Cdk ${ }^{f / / f l} L y 5.1$, and Cdk8 ${ }^{\Delta / \Delta}$ Ly5.2 cells; $\beta$ actin served as loading control. Levels of significance were calculated using $\mathbf{b}, \mathbf{d}, \mathbf{e}, \mathbf{f}$ unpaired $t$-test, $\mathbf{i}$ Mann-Whitney, and $\mathbf{c}$ Kruskal-Wallis test followed by Dunn's test; data represent means \pm SD $\left({ }^{\star \star} p<0.01\right)$. Source data are provided as a Source Data file

pro-B cells that consistently stain positive for B220, CD19, and CD43, and negative for the maturation markers IgM and IgD (Fig. 3c). BCR-ABL1 $185+$ and $\mathrm{v}-\mathrm{ABL}^{\mathrm{p} 160+}$-transduced $C d k 8^{\Delta / \Delta}$ Vav-Cre cell lines showed higher frequencies of apoptotic cells (Fig. $3 \mathrm{~d}$ and Supplementary Fig. 3c) that were also evident in growth curves; the numbers of v-ABLp160+C $C d k 8^{\Delta / \Delta} V a v-C r e$ cells rose significantly slower than those of the control group (Supplementary Fig. 3d). Consistently, the number of cells in the $\mathrm{SubG}_{1}$ phase increased upon loss of CDK8 (Fig. 3e and Supplementary Fig. 3e).

The enhanced apoptosis and reduced proliferation of CDK8-deficient cells in vitro may be compensated in vivo by the microenvironment. We transplanted BCR-ABL1p185+ or $\mathrm{v}-\mathrm{ABL} \mathrm{L}^{\mathrm{p} 60+}$ transformed $C d k 8^{\Delta / \Delta}$ cell lines into NSG mice. Leukemia latency of BCR-ABL1 ${ }^{185+}$ and v-ABLp160+ transformed $C d k 8^{\Delta / \Delta}$ cells was significantly increased compared to wild-type cells (Fig. 3f and Supplementary Fig. 3f). Analysis of moribund animals unraveled slight differences in disease phenotypes; although the numbers of WBCs were comparable, the frequency of CD19+ cells containing the transformed cell population in the BM was significantly increased upon CDK8 loss (BCR-ABL1 ${ }^{\text {p185+ }}$ ) (Fig. $3 g$ and Supplementary Fig. 3g). The absence of CDK8 was confirmed by immunoblotting of ex vivo-isolated BCR-ABL1P185+- and v-ABL $160+$-transduced cells (Fig. $3 \mathrm{~h}$ and Supplementary Fig. $3 \mathrm{~h}$ ). The prolonged disease latency of the $C d k 8^{\Delta / \Delta} V a v$-Cre-transplanted cohort does not result from differences in homing to the BM, as there were no significant differences between BCR-ABL1p185+ CDK8-expressing and BCR-ABL1 $185+C d k 8^{4 / \Delta}$ Vav-Cre cells (Fig. 3i). Expression of a CDK8 kinase-dead mutant (D173A) in leukemic $C d k 8^{\Delta / \Delta} \mathrm{Vav}$-Cre cells accelerated disease development, which indicates that CDK8 exerts its effect in a kinase-independent manner (Supplementary Fig. 3i, j). The data suggest that CDK8 is largely dispensable for the initiation of BCR-ABL1 ${ }^{185+}$ or $\mathrm{v}$-ABL $160+\mathrm{B}-\mathrm{ALL}$, but instead may play an important part in disease maintenance by controlling cell survival.

CDK8 is required for maintenance of $B C R-A B L 11^{p 185+}$ leukemia. To investigate the immediate consequence of CDK8 loss in leukemic cells, we created $C d k 8^{f l f l} M \times 1 C r e$ BCR-ABL1 $185+$ cell lines and deleted $C d k 8$ at specific time points by IFN- $\beta$ (Fig. $4 \mathrm{a}$ ). Immunoblotting confirmed that IFN- $\beta$ administration deleted $C d k 8$ until day 7 , after which multiplication of the few remaining Cdk8-positive (non-deleting) cells gave rise to a weak signal for CDK8 (Fig. 4b). Whereas wild-type BCR-ABL1P185+ cells were only marginally affected by IFN- $\beta$ stimulation, the percentage of AnnexinV-positive cells was significantly over 7 days upon loss of CDK8 (Fig. 4c). Cell cycle analysis on day 3 after addition of IFN$\beta$ revealed a significant increase of cells in $\mathrm{SubG}_{1}$ and a reduction of cells in the $S-$ and $G_{2}$ phases (Fig. $4 d$ ). To test the effects of deletion of $C d k 8$ in vivo, we transplanted BCR-ABL1p185+ wild type and $C d k 8^{f l f l} M x 1 C r e$ into NSG mice (Fig. 4e). Deletion of $C d k 8$ resulted in a significant survival benefit (Fig. $4 \mathrm{f}$ ). Immunoblotting of the leukemic cells ex vivo revealed residual CDK8 expression in the leukemic compartment, representing heterozygous deleters or repopulating BCR-ABL1 ${ }^{\mathrm{p} 185+} \mathrm{Cdk} 8^{f l f l} \mathrm{M} \times 1 \mathrm{Cre}$ cells that managed to avoid deletion (Fig. $4 \mathrm{~g}$ ). These non-deleters caused the fatal leukemia with a significant increase in circulating WBCs but a decreased frequency of $\mathrm{CD} 19^{+}$cells in the spleen (SPL) (Fig. 4h, i). To follow the kinetics of leukemic cells, we again injected BCR-ABL1 $185+C d k 8^{f l / f l} M x 1 C r e$ into NSG mice and found a pronounced decrease in the BM upon poly(I:C) treatment after 17 days. Leukemic cells increased again thereafter, indicating a selection of non-deleters that finally induce disease (Supplementary Fig. 4a, b). These experiments using inducible deletion led us to conclude that BCR-ABL1 $185+$ cells depend on CDK8.

Kinase inhibition fails to mimic the effects of $C d k 8$ deletion. Various small-molecule inhibitors have been developed to target CDKs in cancer. To further investigate whether CDK8's kinase activity accounts for the substantial effects in leukemogenesis, we applied the CDK8/CDK19 inhibitors Senexin B and MSC (MSC2530818). The efficiency of the inhibitors was confirmed by reduction of STAT $1^{\text {S727 }}$ phosphorylation, a known CDK8 kinase substrate (Fig. 5a) ${ }^{24}$. The reduced phosphorylation was accompanied by a lower induction of STAT1 target genes (Mx1, Stat1, Tap1, Gbp2, Irf1, and Ido1) upon IFN- $\beta$ stimulation (Supplementary Fig. 5a). Despite reduction of pSTAT1 ${ }^{\mathrm{S} 727}$ and its target genes, there was no significant change in the frequency of apoptotic cells in BCR-ABL1 $185+C d k 8^{f l / f l}$ and $C d k 8^{\Delta / \Delta}$ Vav-Cre cell lines upon treatment with inhibitor (Fig. 5b). The $\mathrm{IC}_{50}$ values for Senexin B were almost identical, irrespective of the presence of CDK8 (Supplementary Fig. 5b). We investigated these differences by RNA sequencing (RNA-seq), comparing four individually derived BCR-ABL1 $185+C d k 8^{\Delta / \Delta} V a v-C r e$ cell lines with four BCR-ABL1p185+ $C d k 8^{f l / f l}$ cell lines in the absence and in the presence of a kinase inhibitor (Senexin B). The absence of CDK8 protein resulted in 103 upregulated and 56 downregulated genes in BCR-ABL1 $185+C d k 8^{\Delta / \Delta}$ Vav-Cre compared with control cell lines. In contrast, Senexin B had only minor effects and affected the transcription of only six genes (Fig. $5 \mathrm{c}$ and Supplementary Fig. 5c). CDK8 deletion is thus not recapitulated by inhibition of CDK8 kinase activity in leukemogenesis.

CDK8 regulates the $m$ TOR pathway. We used gene set enrichment analysis (GSEA) to identify pathways that are perturbed in the absence of CDK8 $\left(C d k 8^{\Delta / \Delta} \mathrm{Vav}-\mathrm{Cre}\right)$. GSEA analysis 
a
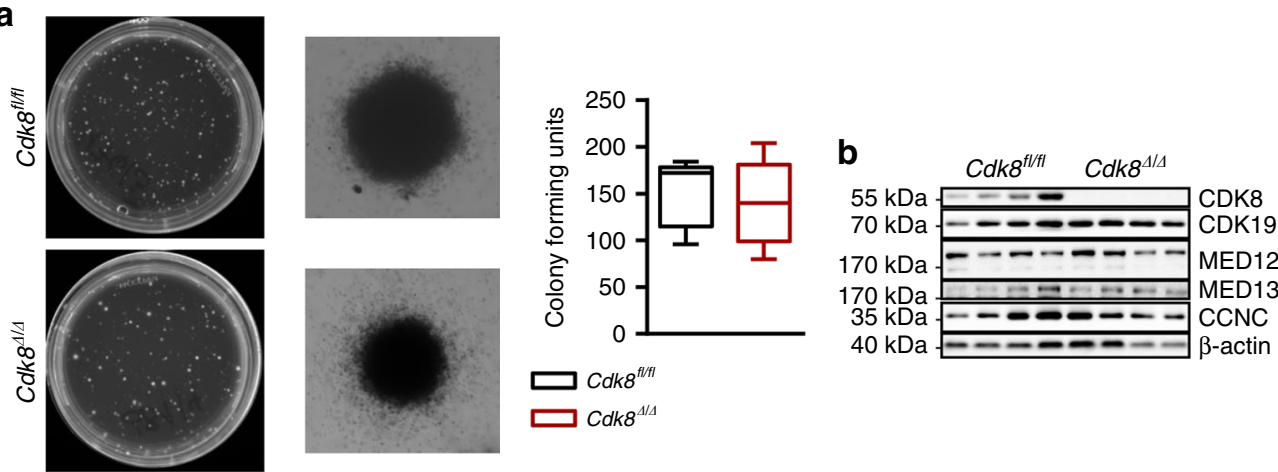

\section{$\square C d k 8^{t / t h t}$ \\ $\square \mathrm{Cdk} 8^{4 / 4}$}

C

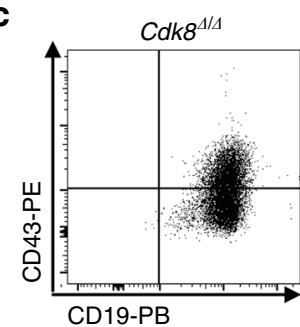

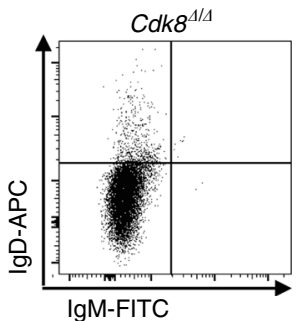

Marker

$\mathrm{CD} 43^{+} \mathrm{CD} 19^{+}$

$\mathrm{CD} 43^{-} \mathrm{CD} 19^{+}$

$\lg \mathrm{D}^{-} \lg \mathrm{M}^{-}$

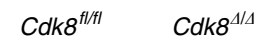

$49.6 \pm 8.2 \quad 44.2 \pm 5.2$

$50.3 \pm 8.2 \quad 55.7 \pm 5.8$

$96.0 \pm 0.9 \quad 94.0 \pm 3.2$ d
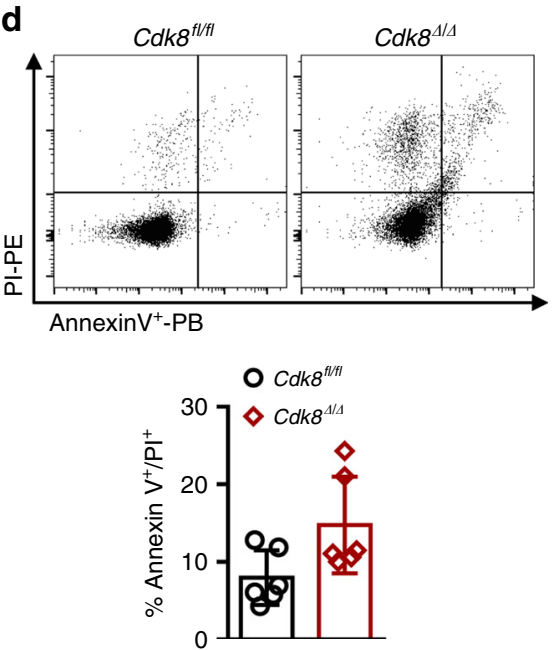
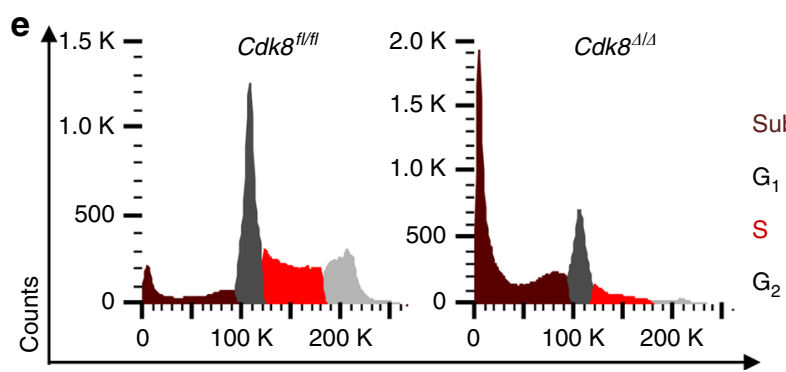

Propidium iodide-PE

f

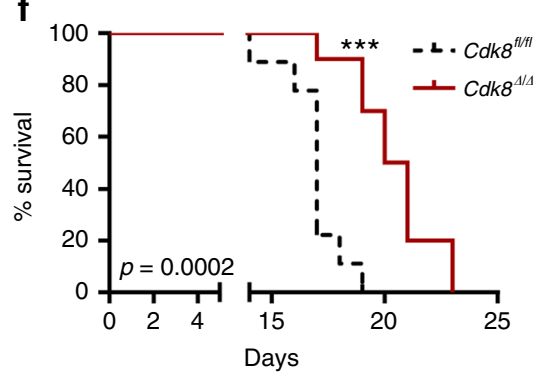

g
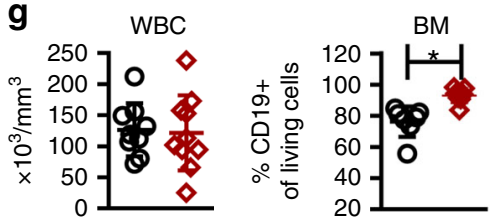

O $C d k 8^{f / f t}$ $\diamond \mathrm{Cdk} 8^{4 / 4}$

h

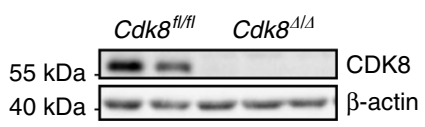

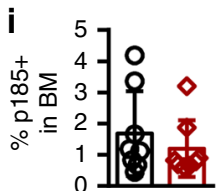

identified downregulation of 14 pathways, including several prominent signaling pathways involved in BCR-ABL1-driven disease. Among the top hits, we found "tumor necrosis factor- $\alpha$ signaling via nuclear factor- $\kappa \mathrm{B}(\mathrm{NF \kappa B})$ ", "mammalian target of rapamycin complex 1 (mTORC1) signaling," and "phosphoinositide 3-kinase (PI3K) AKT mTOR signaling" (Fig. 6a).
A complete list of deregulated pathways is provided in Supplementary Table 1. Confirmation was provided by the EnrichR program (Supplementary Fig. 6a and Supplementary Table 2), which validated the deregulation of 18 genes (Supplementary Fig. 6b). Phosphorylation of key players in the mTOR pathway $\left(\mathrm{pS}^{\mathrm{S} 240 / 244}, \mathrm{p} 4 \mathrm{E}-\mathrm{BP}-1^{\mathrm{T} 37 / 46}\right.$, and $\left.\mathrm{pAKT}^{\mathrm{S} 473}\right)$ was reduced in 
Fig. 3 CDK8 is not required for initial BCR-ABL1p185+ transformation. a BCR-ABL1p185-induced colony formation of Cdk8fl/fl and Cdk8 $8^{\Delta / \Delta V a v-C r e ~ B M ~ c e l l s ~ i n ~}$ growth-factor-free methylcellulose. The second panel shows single-colony pictures of each phenotype. Summary of colony-formation assays. Center value represents median, the box 25 th to 75 th percentiles and whiskers min to max ( $n=4$ per genotype; each performed in duplicates). b CDK8, CDK19, CCNC, MED12, and MED13 protein levels in BCR-ABL1p185+ Cdk8fl/fl and BCR-ABL1p185+ Cdk8 $8^{\Delta / \Delta}$ Vav-Cre cell lines (immunoblotting). $\beta$-Actin served as loading control. c Representative FACS blots of B-cell marker staining: B220 ${ }^{+}$gated BCR-ABL1p185+ Cdk8 $8^{\Delta / \Delta}$ Vav-Cre cells with CD43, CD19, IgD, and IgM. Table below indicates frequencies of indicated markers for BCR-ABL1p185+Cdk8fl/fl and BCR-ABL1p185+Cdk8 $8^{\Delta / \Delta}$ Vav-Cre cell lines $(n=4$ per genotype). $\mathbf{d}$ FACS profile of an AnnexinV/PI staining of BCR-ABL1p185+ Cdk8fl/fl and BCR-ABL1p185+ Cdk8 $8^{\Delta / \Delta V a v-C r e ~ c e l l ~ l i n e s ~}(n=6$ per genotype). e PI cell cycle staining of BCR-ABL1p185+ Cdk $8^{f / / f l}$ and BCR-ABL1p185+ $C d k 8^{\Delta / \Delta V a v-C r e ~ c e l l ~ l i n e s . ~ T h e ~ e x p e r i m e n t ~ w a s ~ p e r f o r m e d ~ i n ~ t e c h n i c a l ~ d u p l i c a t e s ; ~ d a t a ~ f r o m ~ o n e ~ o u t ~ o f ~ t h r e e ~}$ independent experiments are depicted. Table contains frequencies of cells in individual phases of the cell cycle $(n=3$ per genotype). $\mathbf{f}$ BCR-ABL1p185+ $C d k 8^{f l / f l}$ and BCR-ABL 1 p185 $+C d k 8^{\Delta / \Delta V a v-C r e ~ c e l l s ~ w e r e ~ i n j e c t e d ~ i n t r a v e n o u s l y ~(i . v .) ~ i n t o ~ n o n-i r r a d i a t e d ~ N S G ~ m i c e ~(2500 ~ c e l l s ~} /$ mouse, $n=9$ mice received BCR-ABL1p185+ Cdk $8^{f / f l}$ and 10 mice BCR-ABL1 $185+C d k 8^{\Delta / \Delta}$ Vav-Cre cells, 3 independent cell lines per genotype were injected). Survival curves of recipients are depicted (median survival of $C d k 8^{f l / f l}$ and $C d k 8^{\Delta / \Delta}$ Vav-Cre cohorts: 17 days and 21 days). $\mathbf{g}$ White blood cell count (WBC) ( $n=9$ Cdk $8^{f l / f l} ; n$

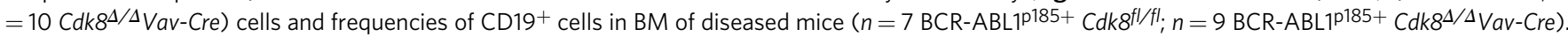

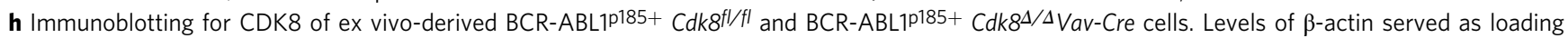
control. i Homing assay of BCR-ABL1p185+ Cdk8fl/fl vs. BCR-ABL1p185+ Cdk8 $8^{\Delta / \Delta}$ Vav-Cre cells. Bar diagram show percentages of BCR-ABL1p185+ cells in the BM $18 \mathrm{~h}$ post injection. Asterisks denote statistical significances as determined by an $\mathbf{a}, \mathbf{c}, \mathbf{e}, \mathbf{g}(\mathrm{WBC})$ unpaired $t$-test, $\mathbf{d}, \mathbf{g}$ (BM), i Mann-Whitney, or a f log-rank test; data represent means \pm SD $\left({ }^{\star} p<0.05 ;{ }^{\star \star \star} p<0.001 ;{ }^{\star \star \star \star} p<0.001\right)$. Source data are provided as a Source Data file

CDK8-deficient cells (Fig. 6b, c). We confirmed the involvement of CDK8 in the regulation of signaling pathways with commercially available inhibitors of the mTOR, PI3K, and NFkB pathways. $\mathrm{IC}_{50}$ levels were determined in BCR-ABL1 $185+C d k 8^{f l / f l}$ and BCR-ABL1 $185+C d k 8^{\Delta / \Delta} V a v-C r e$ cell lines (Fig. 6d). Loss of CDK8 was associated with a $>20$-fold enhanced susceptibility to the two mTOR inhibitors Torin1 and Everolimus. The combined inhibition of PI3K and mTOR (by BEZ235) was threefold more effective in $C d k 8$-positive cell lines, presumably because of feedback loops. Sensitivity to inhibition of the NFKB pathway (by Bay11) was little altered by the loss of CDK8 and the effects of inhibition of PDK1/Akt, Flt3/PIM, JAK1, and JAK2 (by Ruxolitinib), and of PI3K $\delta$ (by Cal101) were also independent of the presence of CDK8. The absence of CDK8 renders cells slightly more susceptible to inhibition of CDK9 (NVP-2) or CDK7 (THZ1 ), although the IC50's are well above the published ranges for specific effects so the results presumably reflect general toxicity (Fig. 6d and Supplementary Fig. 6c). The findings confirm that CDK8 has a role in the mTOR signaling pathway.

Chemical CDK8 degradation cooperates with mTOR inhibition. We analyzed publicly available data from large cohorts of ALL ( $n=203$; TARGET study) and AML $(n=179$; The Cancer Genome Atlas (TCGA) study) patients for a potential link between CDK8 and members of the mTOR pathway. Data on AML patients were included, as CDK8 has been implicated in the disease $\mathrm{e}^{31}$. In both diseases, we found a significant correlation of CDK8 with members of the mTOR pathway including CREB1, TSC1, mTOR, PIK3CD, ZZI1, DEPTOR, SOS1, RRAGB, LAMTOR5, PIK3CB, and PTEN (Fig. 7a, b). Furthermore, CDK8 and members of the mTOR pathway are correlated in a number of other cancers including thyroid carcinoma, thymoma, prostate adenocarcinoma, and liver hepatocellular carcinoma (Supplementary Fig. 7a).

To evaluate the therapeutic potential of our findings, we targeted CDK8 alone or in combination with mTOR inhibition in human leukemic cells (cell lines or primary samples). As inhibition of CDK8 kinase activity does not mimic the effects of CDK8 knockdown in mice, we used chemical-induced protein degradation strategy ${ }^{36}$ to deplete the CDK8 protein in human leukemic cell lines. The CDK8 degrader JH-XI-10-02 selectively degrades $\mathrm{CDK} 8^{37}$. In addition, medical chemistry optimization of Torin1, an inhibitor of mTOR, which also affects CDK8 kinase activity $\left(\mathrm{IC}_{50} \text { of } 159 \mathrm{nM}\right)^{38}$, resulted in the development of THZ455 , a more potent inhibitor of CDK8 $\left(\mathrm{IC}_{50}=5 \mathrm{nM}\right)$. By linking THZ4-55 with thalidomide, a ligand that can recruit the E3 ligase cereblon, we generated a bivalent CDK8 degrader, YKL-06-101, which can also inhibit mTOR (Fig. 7c). We tested Senexin B, JHXI-10-02, and YKL-06-101 in human BCR-ABL1 ${ }^{+}$(BV173, Nalm1, TOM-1, and Z119) and BCR-ABL1- (BL41, Raji, Ramos, and REH) cells. Degrader JH-XI-10-02 and Senexin B treatment induces a proliferation arrest at $\mathrm{IC}_{50}$ values above $10 \mu \mathrm{M}$. In contrast, the bivalent degrader YKL-06-101 stops proliferation of BCR-ABL1 ${ }^{+}(\mathrm{Z119}, \mathrm{BV} 173)$ and of BCR-ABL1 ${ }^{-}$cells $(\mathrm{REH})$ at concentrations below $1 \mu \mathrm{M}$ (Fig. $7 \mathrm{~d}$ ). Growth arrest is observed after $48 \mathrm{~h}$ (Fig. $7 \mathrm{~d}$ ) and is followed by induction of apoptosis and a significant increase in Annexin $\mathrm{V}^{+} / 7 \mathrm{AAD}^{+}$cells (Fig. $7 \mathrm{e}, \mathrm{h}$ and Supplementary Fig. 8a). Degradation of CDK8 but not of mTOR was verified by western blotting (Fig. 7f, $g$ and Supplementary Fig. 8b). The reduced phosphorylation of $\mathrm{S}^{\mathrm{S} 240 / 244}$, indicative of mTOR inhibition, confirms the dual function of the YKL-06-101 degrader (Fig. 7f, g and Supplementary Fig. 8b). Levels of CDK19, CCNC, MED12, and MED13 remained unaltered, irrespective of whether the CDK8 protein was degraded or its kinase activity was inhibited (Supplementary Fig. 8c, d). We verified the synergistic effects of CDK8 degradation and $\mathrm{mTOR}$ inhibition by treating cells simultaneously with the CDK8 degrader JH-XI-10-02 and Torin 1 . Incubation of BCR-ABL1 ${ }^{+} \mathrm{Z} 119$ cells with $1 \mu \mathrm{M} \mathrm{JH}-\mathrm{XI}-$ 10-02 and $10 \mathrm{nM}$ Torin 1 caused significantly more apoptosis than single treatments, confirming that the effects result from the simultaneous degradation of CDK8 and the inhibition of mTOR (Fig. 7i).

Treatment of primary ALL patient samples with JH-XI-10-02 and YKL-06-101 confirmed the efficacy of YKL-06-101 but not of JH-XI-10-02 alone to induce apoptosis. YKL-06-101 reduced cell viability in 5 out of 12 patient samples, whereas JH-XI-10-02 had no effect (Fig. $7 \mathrm{j}$ and Supplementary Table 3 ). The result strongly suggests that combining CDK8 degradation with mTOR inhibition may represent a therapeutic approach for a subset of ALL patients.

\section{Discussion}

CDK8 has attracted much attention over the last years as potential target for cancer therapy ${ }^{39}$. Small-molecule inhibitors that block CDK8 kinase activity have been shown to be effective in $\mathrm{AML}^{31}$, melanoma ${ }^{27}$, breast ${ }^{29}$, prostate ${ }^{28}$, and colon cancer models ${ }^{39,40}$. We describe a kinase-independent function of CDK8 in hematopoietic tumors and suggest that the proteasomeinduced degradation of CDK8 in combination with inhibition of mTOR might represent an addition to the therapeutic armory.

CDKs are present at high levels in hematopoietic neoplasms. Although murine leukemia cells (BCR-ABL1p185+) contain high 
a

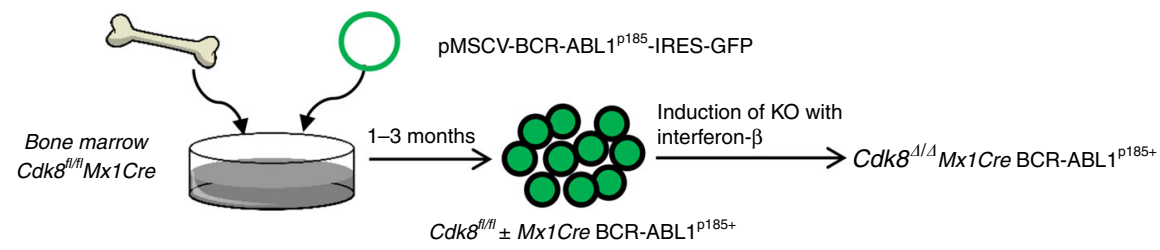

b
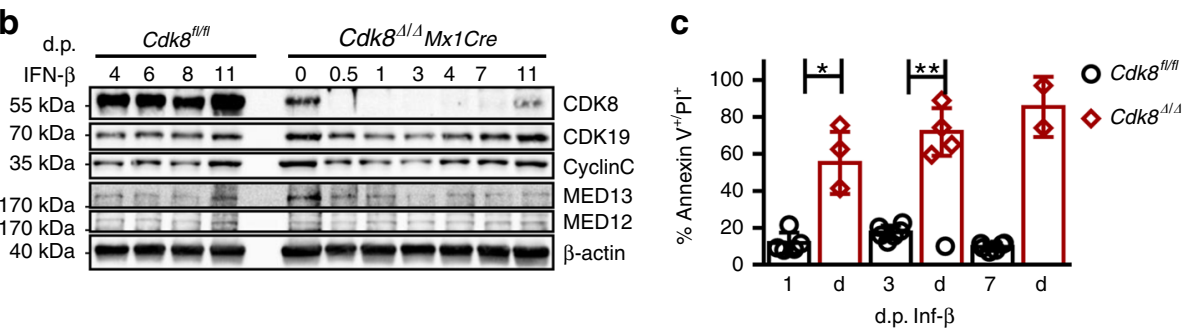

d
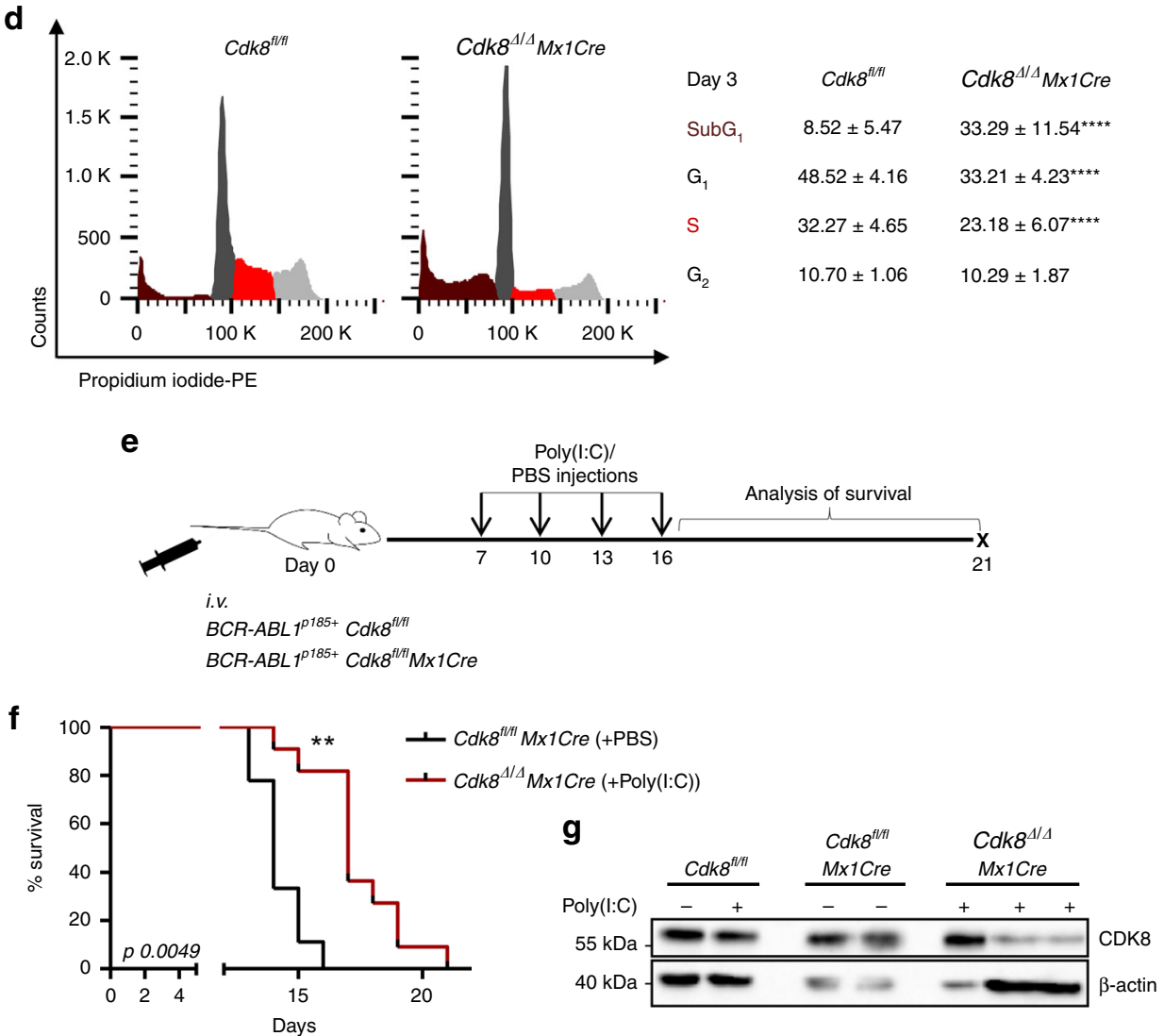

h
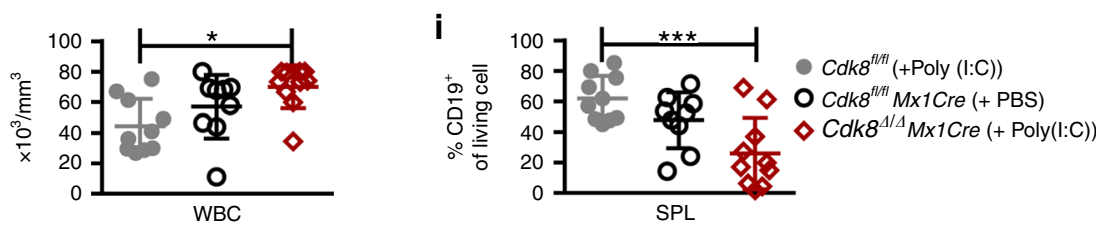

levels of CDK6, CDK7, CDK8, CDK9, and CDK19, knockdown of CDK6, CDK7, CDK9, or CDK19 has little effect on cell survival and proliferation in an inducible system. In contrast, deletion of CDK8 has a dramatic effect. Stable knockdown experiments recapitulated the results with inducible shRNAs, although we were not able to generate CDK7- or CDK8-deficient cell lines. The apparent discrepancy between the short- and long-term effects of CDK7 knockdown can be explained by speculating the existence of a stable factor that depends on CDK7; further experiments will be required to test this notion. The pronounced anti-proliferative effects of $C d k 8$ deletion in murine leukemia cells were unexpected and indicate the unique role of CDK8 downstream of BCR-ABL1 ${ }^{\text {185+ }}$ in murine B-ALL. The effect is at least partially independent of the mediator as the deletion of CCNC or MED13 was well tolerated. The pronounced effects of deleting MED12 may be explained by the requirement for MED12 in 
Fig. 4 CDK8 is required for maintenance of BCR-ABL1p185+ leukemia. a Generation of stable BCR-ABL1p185+ Cdk8fl/fl and BCR-ABL1p185+ Cdk8fl/flMx1Cre cell lines. Scheme depicts experimental setup of data shown in b-d. b Efficiency of CDK8 deletion and protein levels of CDK19, CCNC, MED12, and MED13 at indicated time points post interferon- $\beta$ (IFN- $\beta$ ) administration (immunoblotting). Levels of $\beta$-actin served as a loading control. $\mathbf{c}$ Proportions of AnnexinV ${ }^{+}$cells in BCR-ABL1p185+ Cdk8fl/fl or BCR-ABL1p185+ Cdk8 ${ }^{\Delta / \Delta}$ Mx1Cre cell lines 1,3 , and 7 days after administration of IFN- $\beta$ (d.p. IFN- $\beta$ : days post IFN- $\beta ; n=6$ BCR-ABL1p185+Cdk8 fl/fl; $n=4$ BCR-ABL1p185+ Cdk8 $8^{\Delta / \Delta} M x 1 C r e$, three independent experiments). d Representative PI cell cycle staining of BCR-

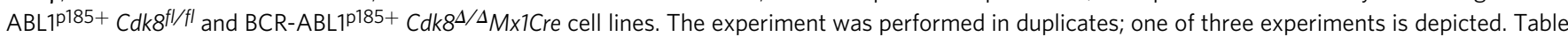
indicates frequencies of cells in individual phases of the cell cycle $(n=3$ per genotype, measured in duplicates). e Scheme depicting experimental setup of in vivo experiment. $\mathbf{f}$ Kaplan-Meier shows survival curves of non-irradiated NSG mice that have received BCR-ABL1 $185+C d k 8^{f l / f l}$ or BCR-ABL1p185+

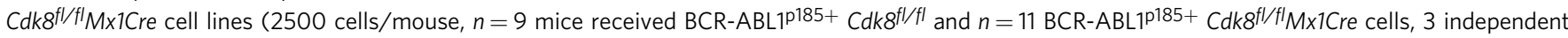
cells lines per genotype were injected). $\mathbf{g}$ Immunoblotting for CDK8 of ex vivo-derived BCR-ABL1p185+ cell lines ( \pm poly(I:C) injections). Levels of HSC70 served as a loading control. $\mathbf{h}$ White blood cell count (WBC) of mice on day of terminal disease (analysis). i Summary of frequencies of CD19+ cells in spleens (SPL) of diseased mice, $n=10$ per genotype. Asterisks denote statistical significances as determined by $\mathbf{d}$ unpaired $t$-test, $\mathbf{c}, \mathbf{h}$ Mann-Whitney, f log-rank test, or i one-way ANOVA followed by Tukey's test; data represent means \pm SD $\left({ }^{\star} p<0.05 ;{ }^{\star \star} p<0.01 ;{ }^{\star \star \star} p<0.001\right)$. Source data are provided as a Source Data file

hematopoietic stem cell homeostasis: Med12flfl Vav1-Cre animals die within 2 weeks of birth with severely reduced $\mathrm{BM}$ and thymus cellularity ${ }^{41}$.

CDK8 does not influence initial transformation by the BCR$\mathrm{ABL} 1 \mathrm{p}^{185+}$ oncogene but is indispensable for the maintenance of established leukemic cell lines. This effect might stem at least in part from CDK8's role in the phosphorylation of STAT5, a key factor for leukemia maintenance $22,24,42$. Similar to STAT5, CDK8 is essential for the survival of leukemic cells, making it a potential therapeutic target. Deletion of $C d k 8$ after disease onset is associated with reduced viability of leukemic cells in vitro and with prolonged survival of the mice in vivo. An additional benefit from targeting $\mathrm{CDK} 8$ might arise from the fact that $\mathrm{CDK} 8$ represses the natural killer (NK) cell-dependent surveillance of hematopoietic tumors ${ }^{23}$. Deletion of CDK8 in NK cells enhances their cytotoxicity and prolongs the survival of mice suffering from BCR-ABL1 ${ }^{+}$leukemia ${ }^{43}$. Degrading CDK8 would thus kill two birds with one stone: it would inhibit the survival of leukemic cells, while enhancing NK cell cytotoxicity.

A pre-requisite for drug development is the existence of a sufficiently large therapeutic window-any therapeutic strategy must harm transformed cells, while sparing their healthy counterparts. Although $\mathrm{CDK} 8$ is absolutely required for the preimplantation of the embryo, $C d k 8$ deletion is well tolerated in adult mice. It does not seem to interfere with homeostasis of hematopoietic organs, irrespective of whether it is performed by means of MxlCre or by Vav-Cre, and has no impact on the viability or functionality of the HSC. Our findings are consistent with previous work, in which deletion of $C d k 8$ in adult mice had no significant effect ${ }^{19}$. It is possible that CDK19, the paralog of CDK8, can compensate for the loss of CDK8 in the adult organism in non-transformed tissues, as proposed in $\mathrm{NK}^{43}$ and prostate cancer cells ${ }^{28}$.

Despite its involvement in the transcription machinery (it associates with the mediator complex), CDK8 appears to have some functions that are context-specific. In AML cell lines, inhibition of CDK8/CDK19 kinase activity upregulates SEassociated genes with tumor suppressor and lineage-controlling functions, thereby exerting anti-leukemic effects ${ }^{31}$. No such effects were seen in HCT116 colon cancer cells ${ }^{31}$ and we do not find them in B-ALL cells. Further studies are required to identify the precise mechanism by which CDK8 influences leukemogenesis. As knockdown of MED13 and CCNC is well tolerated, at least some of the effects are independent of the mediator complex.

Although CDK8 was initially discovered as a kinase that regulates transcription, we provide evidence that it has a function that is independent of its kinase activity: inhibition of the CDK8 kinase activity does not consistently mirror the effects of Cdk8 gene knockdown. Kinase inhibition with Senexin B or MSC causes only minor effects on transcription, whereas the deletion of CDK8 significantly affects transcriptional responses and critical signaling pathways in BCR-ABL1 ${ }^{\mathrm{p}^{185}+}$-driven leukemia. This finding is consistent with work in HCT116 colon cancer cells, in which inhibition of CDK8 kinase activity induces weak anti-proliferative responses, whereas gene deletion has pronounced effects $5,25,31,44$. Deletion of $C d k 8$ (but not inhibition of its kinase activity) leads to reduced levels of mTOR signaling, which has been implicated in BCR-ABL1 ${ }^{+}$leukemia ${ }^{45}$. Although loss of CDK8 and mTOR inhibition has synergistic effects, not such interdependence is seen between the loss of CDK8 and PI3K inhibition. This apparently paradoxical result presumably stems from the complex feedback loops and crosstalk between the mTOR and other PI3K-dependent pathways. CDK8 has been suggested to interact with mTOR in the context of lipogenesis ${ }^{46}$ but not in any form of cancer. An analysis of publicly available RNA-seq data from a range of human cancers suggests that CDK8 levels are highly correlated with the levels of members of the mTOR pathway. The mechanism for the interaction remains a matter for conjecture: we have no direct evidence for or against a physical interaction between CDK8 and mTOR.

TKIs have revolutionized leukemia therapy. Nevertheless, the prognosis for many types of cancer remains poor and patients face a high risk of acquiring resistance-mediating mutations. Specific protein degradation represents a recent mechanism to target proteins independent of their enzymatic activity. We have investigated the potential application of CDK8 degraders, testing the effects of two structurally distinct compounds in human leukemic cell lines. Both molecules cause the efficient degradation of CDK8 but only YKL-06-101 is able to block mTOR signaling. The combined effect of degrading CDK8 and inhibiting mTOR significantly increased apoptosis in three human leukemic cell lines. We believe that this strategy holds a great promise for the clinic, as targeting two unrelated signaling pathways will reduce the probability of developing resistance. Our preliminary investigations suggest that CDK8 interacts with the mTOR signaling pathway not only in a subset of leukemia patients but also in a range of solid cancers. We therefore propose that a combinatorial therapy could represent an approach to treat a range of human cancers.

\section{Methods}

Cell culture. The following commercially available cell lines were obtained from American Type Culture Collection (ATCC). Established human B-lymphoid cell lines: RL-7 (ATCC no. CRL-2261), REH (ATCC no. CRL-8286), Daudi (ATCC no. CCL-213), and Ramos (ATCC no. CRL-1596). Transformed human T cell lines: Molt-4 (ATCC no. CRL-1582), Jurkat (ATCC no. TIB-152), Mac2A 47 (RRID: CVCL_H637), and HPB-ALL ${ }^{48}$. K562 (ATCC no. CCL-243) is a welldefined human erythroid leukemia cell line transformed by the BCR-ABL1 oncogene. The following cell lines were kindly provided by Peter Valent and 
a

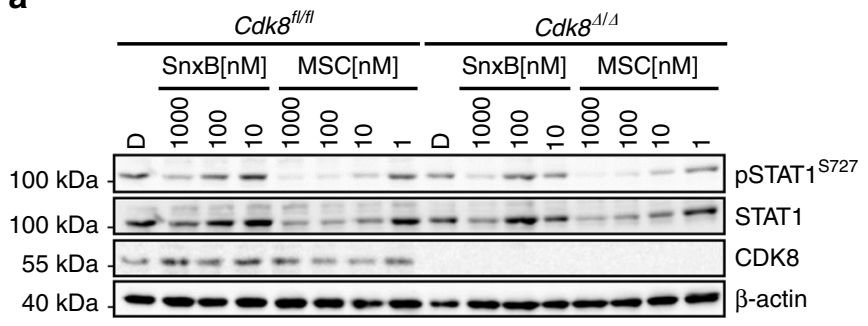

b

$$
\begin{aligned}
& \text { O } C d k 8^{f f f t} \\
& \mathrm{Cdk}^{\mathrm{fl} / 4}
\end{aligned}
$$

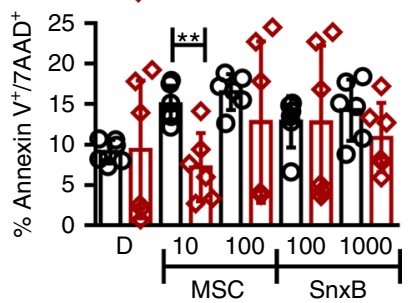

C

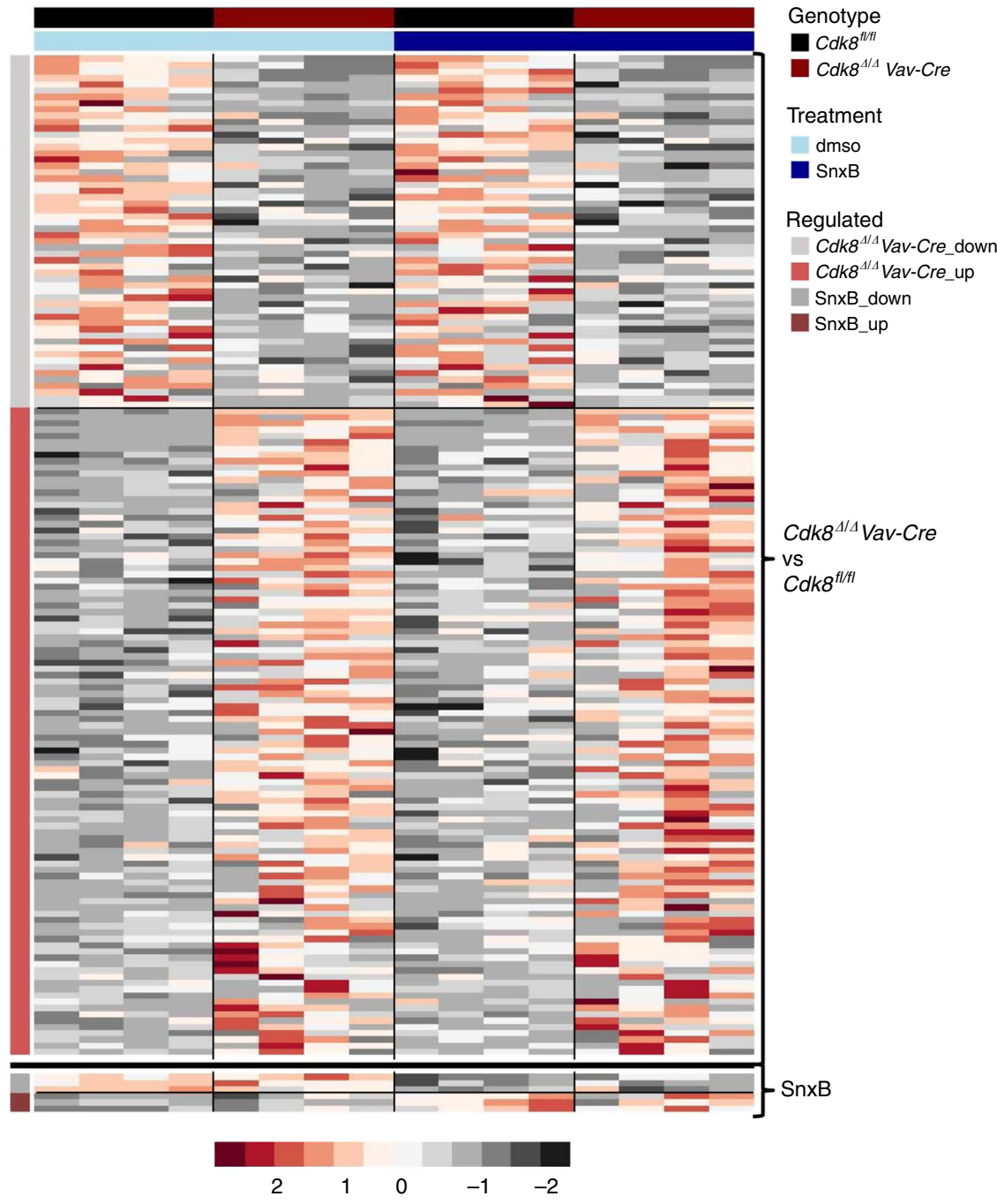

Fig. 5 Kinase inhibition fails to mimic the effects of $C d k 8$ deletion. a Immunoblotting of pSTAT1 5727 of BCR-ABL1p185+ cells after $48 \mathrm{~h}$ incubation with increasing concentrations of MSC or Senexin B (SnxB). Induction of phosphorylation was induced by 30 min IFN- $\beta$ stimulation prior collection. $\beta$-Actin served as a loading control. b AnnexinV/PI staining (after $48 \mathrm{~h}$ ) of BCR-ABL1p185+Cdk8fl/fl and BCR-ABL1p185+ Cdk8 ${ }^{\Delta / \Delta V a v-C r e ~ c e l l ~ l i n e s ~ i n ~ t h e ~ p r e s e n c e ~ o f ~}$ indicated Senexin B or MSC concentrations. DMSO (0.1\%) served as solvent control. Bars represent means \pm SD from two independent experiments $(n=2$ per genotype, measure in triplicates). Asterisks denote statistical significances as determined by unpaired $t$-test $\left({ }^{\star \star} p<0.01\right)$. c Heatmap of 159 differentially expressed genes between BCR-ABL1p185+ Cdk8fl/fl and BCR-ABL1p185+Cdk8 $8^{\Delta / \Delta}$ Vav-Cre cell lines $(n=4$ per genotype). The black line separates the sets of genes, which are differentially regulated upon loss of the entire CDK8 protein (BCR-ABL1 $185+C d k 8^{\Delta / \Delta}$ Vav-Cre cell lines vs. BCR-

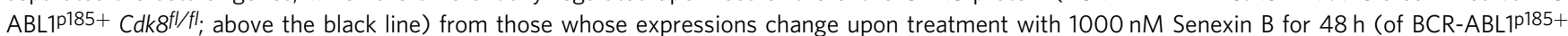
$C d k 8^{f l / f l}$ cell lines) (FDR $<0.1$ ). Colors display centered and scaled $r$-log counts ranging from red to gray (high to low expression). Source data are provided as a Source Data file

purchased from the Leibnitz Institute DSMZ-German Collection of Microorganisms and Cell Cultures; $\mathrm{Ph}^{+}$cell lines: KU812 (RRID: CVCL_0379), TOM-1 (RRID: CVCL_1895), NALM1 (RRID: CVCL_0091), BV173 (RRID: CVCL_0181); $\mathrm{Ph}^{-}$cell lines: BL41 (RRID: CVCL_1087) and Raji (RRID: CVCL_0511) are $\mathrm{Ph}^{-}$. The $\mathrm{Ph}^{+} \mathrm{Z} 119$ (RRID: CVCL_IU88) cell line was kindly provided to J. V. Melo by Zeev Estrov. hMNLs were isolated from peripheral blood samples over a Ficoll gradient. Human leukemia cell lines and different $\mathrm{v}-\mathrm{ABL}{ }^{160+}$, BCR-ABL1P185+ mouse cell lines were cultured in RPMI-1640 (Sigma) supplemented with heat-inactivated fetal calf serum (FCS), $50 \mu \mathrm{M} 2$ mercaptoethanol, and $100 \mathrm{U} / \mathrm{mL}$ penicillin/streptomycin (Sigma). A010 cells (Ab-MuLV producer) and phoenix-ECO (ATCC ${ }^{\circledast 2}$ CRL-3214 $^{\mathrm{TM}}$ ) packaging cells were cultured in Dulbecco's modified Eagle's medium (DMEM) (Sigma) medium 


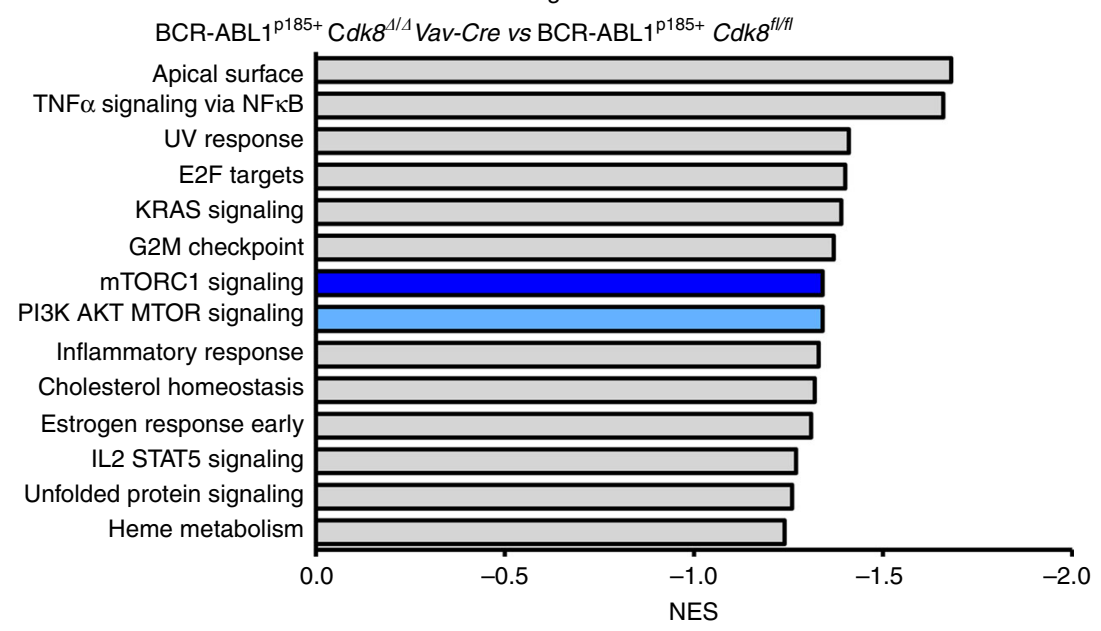

b

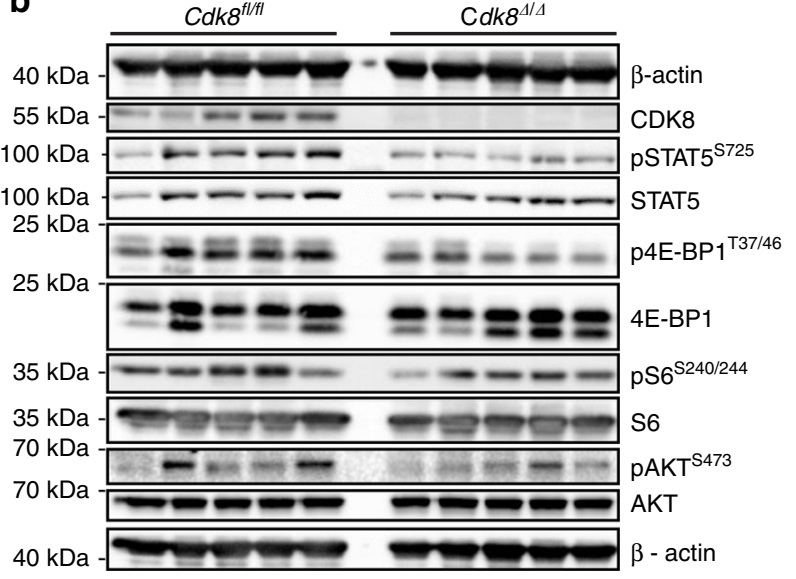

C

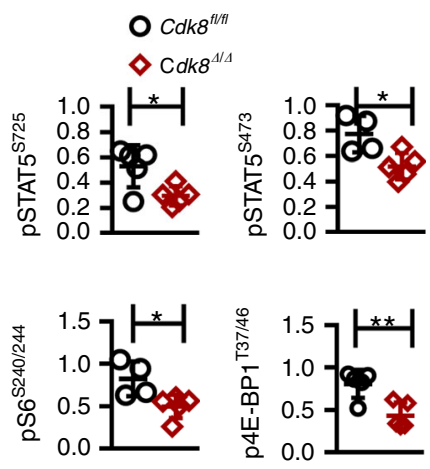

d

$-0 \cdot C d k 8^{t / t / t}$

$\multimap \mathrm{Cdk} 8^{4 / 4}$ Vav-Cre
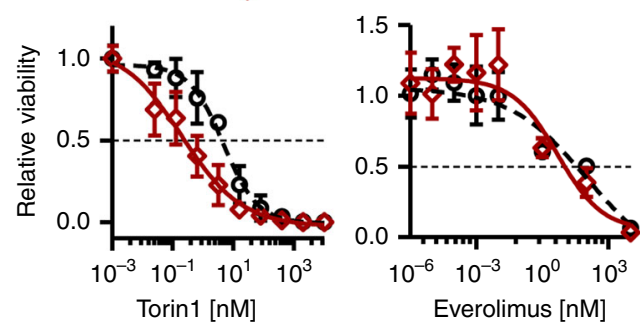

\begin{tabular}{|l|c|c|}
\hline $\mathrm{IC}_{50}[\mathrm{nM}]$ & $\mathrm{Cdk} 8^{\mathrm{flffl}}$ & $\mathrm{Cdk} 8^{\mathrm{A} / 4}$ \\
\hline Torin1 & 4.8 & 0.2 \\
Everolimus & 113 & 5 \\
BEZ235 & 11 & 30 \\
Cal101 & 35 & 23 \\
Bay11 & 3353 & 3779 \\
Ruxolitinib & 457 & 312 \\
PDK1/Akt & 492 & 780 \\
\& Flt3/PIM & & 254 \\
THZ-1 & 543 & 6 \\
NVP-2 & 11 & \\
\hline
\end{tabular}
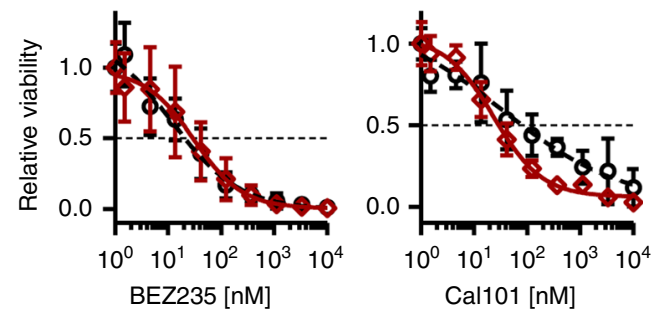

Fig. 6 CDK8 regulates the mTOR pathway. a Gene set enrichment analysis (RNA-seq data) reveals 14 significantly downregulated pathways in BCR-

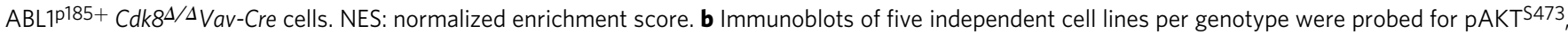
total AKT, pS6 ${ }^{\mathrm{S} 240 / 244}$, total S6, p4E-BPT34/46, and total 4E-BP-1. In addition, pSTAT5 5725 , STAT5, and CDK8 were blotted. $\beta$-Actin served as loading control. c Bar diagrams depict densitometric analysis of signal intensities relative to the loading control (of western blottings shown in b). d Representative dose-response curves for Torin1, Everolimus, BEZ235, and Cal101; mean \pm SD. Table includes IC 50 values of Torin1 (mTORC1 and mTORC2), Everolimus (mTORC1), BEZ235 (PI3K/ATM/ATR and mTOR), Cal101 (PI3Kdelta), Bay11 (NFkB), Ruxolitinib (JAK1 and JAK2), PDK1/Akt and Flt3/Pim dual inhibitor, THZ-1 (CDK7) and NVP-2 (CDK9) on BCR-ABL1p185+ Cdk8fl/fl, and BCR-ABL1p185+ Cdk8 $8^{\Delta / \Delta}$ Vav-Cre cell lines. Data represent the summary of one to three cell lines per genotype in technical triplicates. Asterisks denote statistical significances as determined by an unpaired $t$-test; mean \pm SD $\left({ }^{\star} p<0.05 ;{ }^{\star \star} p<\right.$ 0.01). Source data are provided as a Source Data file 
a

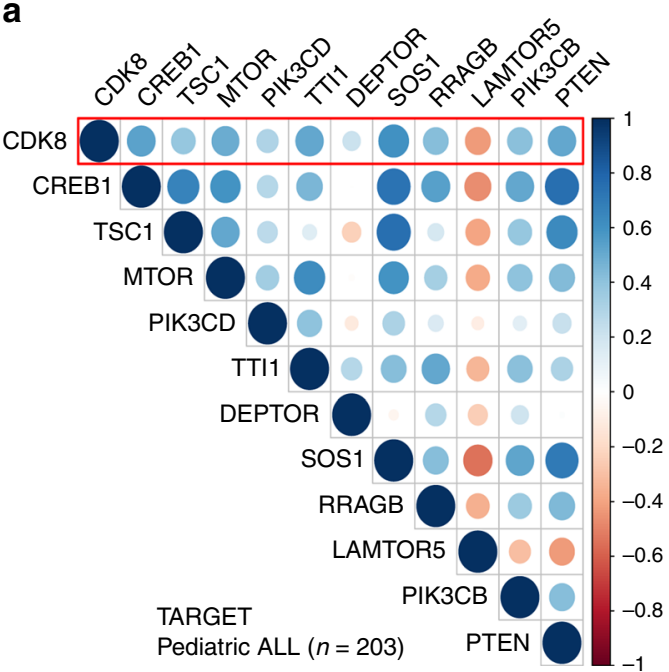

b

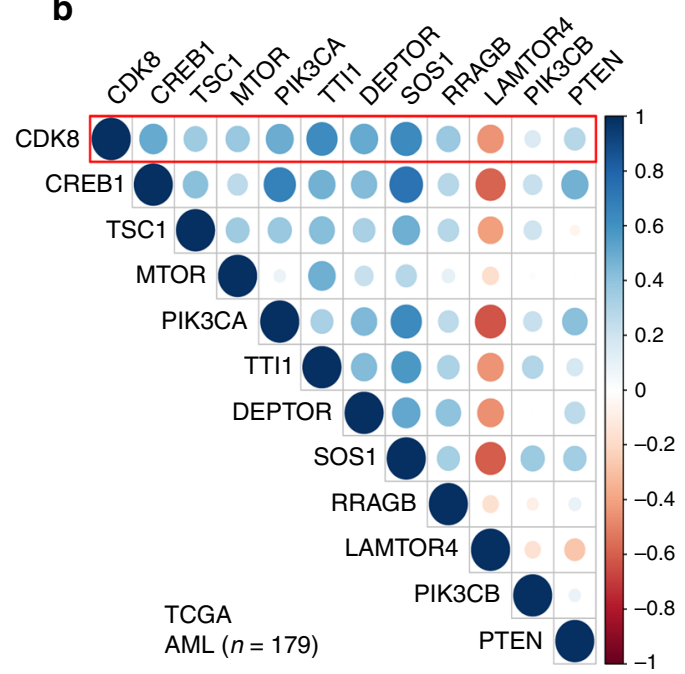

c

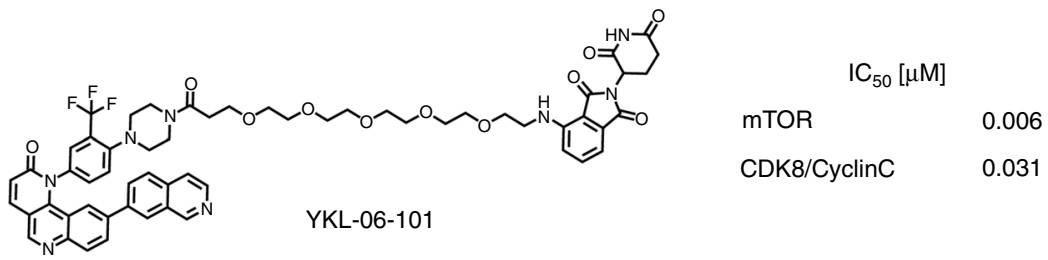

d

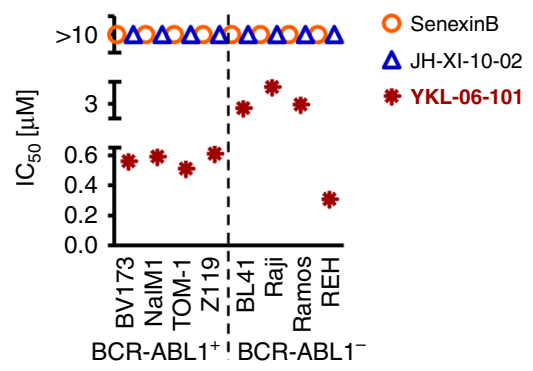

$\mathbf{f}$

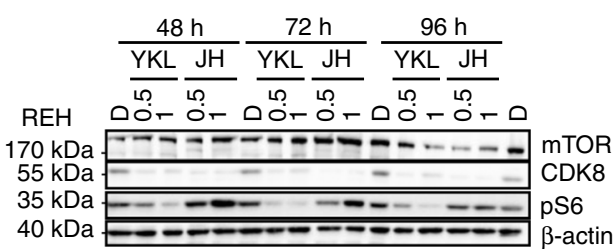

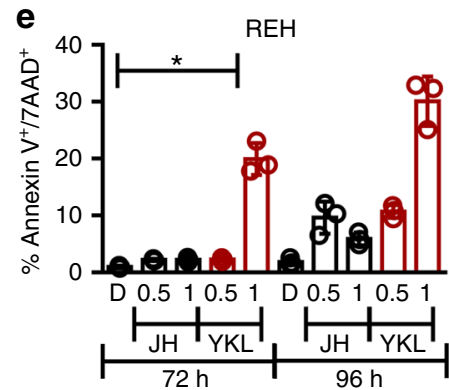

g

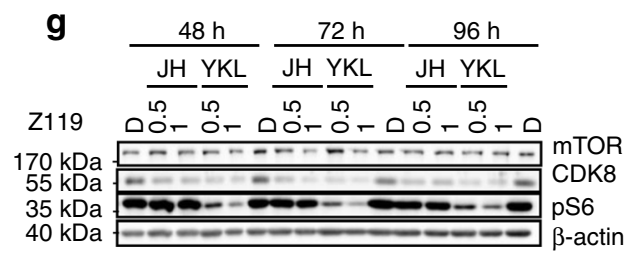

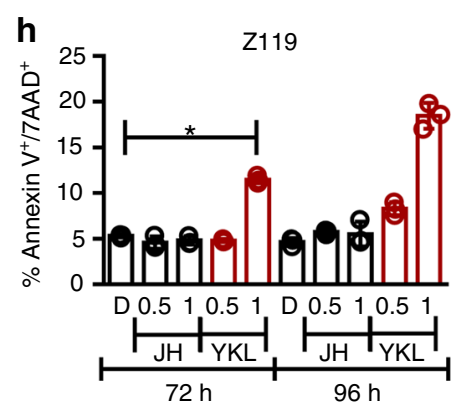

i containing 10\% FCS and $100 \mathrm{U} / \mathrm{mL}$ penicillin/streptomycin. Cell lines are maintained at $37^{\circ} \mathrm{C}\left(5 \% \mathrm{CO}_{2}\right)$ and have been tested regularly for the absence of Mycoplasma.

shRNA knockdowns. For the inducible knockdown experiments, 20 bp shRNAs against mouse CDK8 (Cdk8 mRNA (NM_153599); starting position: 2547), CDK6
(Cdk6 mRNA (NM_009873) starting position: 897), CDK7 (Cdk7 mRNA (NM_009874) starting position: 1145), CDK9 (Cdk9 mRNA (NM_130860) starting position: 2872), CDK19 (Cdk19 mRNA (NM_198164) starting position 1560), CCNC (Ccnc mRNA (NM_016746) starting position: 594), MED12 (Med12 mRNA (NM_021521) starting position: 5755), and MED13 (Med13 mRNA

(NM_001080931) starting position: 9367) were designed based on improved design rules and cloned into microRNA stem (miR-E) in the pSIN-TRE3G-dsRED-miR- 
Fig. 7 Chemical CDK8 degradation cooperates with mTOR inhibition. a Co-expression matrix of CDK8 and members of mTOR/PI3K signaling pathways in samples of 203 pediatric ALL patients (TARGET cohort, RNA-seq) and b 179 AML patients (TCGA AML cohort, RNA-seq). The scale indicates Spearman's correlation coefficients ranging from -1 (red) to 1 (blue). c Structure of degrader YKL-06-101 and biochemically verified IC $C_{50}$ 's for mTOR and CDK8/Cyclin C inhibition. d Thymidine incorporation assay based IC $50^{\prime}$ 's of BCR-ABL1+ (BV173, Nalm1, Tom1, Z119) and BCR-ABL1- (BL41, Raji, Ramos, REH) human leukemic cell lines after $48 \mathrm{~h}$ incubation with increasing concentrations of JH-XI-10-02, YKL-06-101, or Senexin B. e AnnexinV/7AAD staining of REH cells incubated with $0.5 \mu \mathrm{M}$ and $1 \mu \mathrm{M}$ of JH-XI-10-02 or YKL-06-101 for 72 and $96 \mathrm{~h}$. DMSO was used as vehicle control (D: DMSO, $n=3$ ). Immunoblots show mTOR, CDK8, and pS6 after incubation of $\mathbf{f}$ REH cells or $\mathbf{g}$ Z119 cells with $0.5 \mu \mathrm{M}$ and $1 \mu \mathrm{M}$ of JH-XI-10-02 or YKL-06-101 for 48 , 72, and $96 \mathrm{~h}$. DMSO (indicated as D) was used as vehicle control and $\beta$-actin served as loading control. $\mathbf{h}$ AnnexinV/7AAD staining of Z119 cells incubated with $0.5 \mu M$ and $1 \mu \mathrm{M}$ of $\mathrm{JH}-\mathrm{XI}-10-02$ or YKL-06-101 for 72 and $96 \mathrm{~h}$. DMSO was used as a vehicle control (D: DMSO, $n=3$ ). i AnnexinV/7AAD staining of Z119 cells incubated with Torin1, JH-XI-10-02, or both in combination with indicated concentrations for 72 and $96 \mathrm{~h}$. DMSO (indicated as D) was used as a vehicle control. One representative experiment out of three is depicted. $\mathbf{j}$ Bar diagram depicts relative thymidine incorporation of six primary B-ALL patient samples incubated for $48 \mathrm{~h}$ with $1 \mu \mathrm{M}$ of YKL-06-101. Patient \#1 represents a non-responder, patient \#4 was BCR-ABL1+ $\left(\mathrm{Ph}^{+}\right)$, whereas the others were BCR-ABL1 - $\left(\mathrm{Ph}^{-}\right)$. Levels of significance were calculated using Kruskal-Wallis test followed by Dunn's test, data represents means \pm SD $\left({ }^{\star} p<0.05,{ }^{\star \star} p<0.01\right)$. Source data are provided as a Source Data file

E-PGK-Neo (RT3GEN)-inducible retroviral vector ${ }^{49}$. Two vectors containing shRNA against Renilla (Ren.713) and MYC (Myc mRNA (NM_001177352) starting position 1888) served as controls. Exact shRNA sequences are available in the Supplementary Table 4.

BCR-ABL1 $185+$ cell lines, modified to express rtTA3 ${ }^{50}$, were cultured in RPMI-1640 as above with additional $2 \mu \mathrm{g} / \mathrm{mL}$ Puromycin (Invivogen/Eubio). TetOn BCR-ABL1 ${ }^{\text {p185+ }}$ cells were transduced with RT3GEN-based retroviral pseudoparticles supplemented with $7 \mu \mathrm{g} / \mathrm{mL}$ polybrene (Sigma). Transduced cells were selected for 2 weeks in $1 \mathrm{mg} / \mathrm{mL}$ G418 (Invitrogen). Positively selected cells were treated with $0.5 \mu \mathrm{g} / \mathrm{mL}$ doxycycline (Sigma) to induce expression of the dsRed-miR-E cassette. To monitor proliferation and dsRED expression, induced BCR-ABL1 ${ }^{185+}$ cell lines were seeded in 48 -well plates at a concentration of 4000 cells $/$ well $/ \mathrm{mL}$. The cells were incubated at $37^{\circ} \mathrm{C}, 5 \% \mathrm{CO}_{2}$, were counted every $48 \mathrm{~h}$, and were transferred to a plate with fresh media. The percentage of dsRED ${ }^{+}$cells was determined by flow cytometry (FACSCanto II BD Biosystems).

Mouse strains. Conditional C57Bl/6N-Cdk $8^{f l / f l}\left(C d k 8^{\operatorname{tm} l c(E U C O M M) H m g u}\right)^{43}$ were breed to $\mathrm{B} 6 \mathrm{~N}-\mathrm{Tg}(M x 1 \mathrm{Cr} e)^{51}$ and $\mathrm{B} 6 \mathrm{~N}-\mathrm{Tg}(\text { Vav-Cre })^{52} . C d k 8^{f l / f l}, C d k 8^{f l / f l} \mathrm{Mx} 1 \mathrm{Cre}$, Cdk8 $8^{f l f l}$ Vav-Cre, Ly5. $1^{+}\left(C D 45.1^{+}\right), L y 5.1 / 2^{+}\left(\mathrm{CD} 45.1^{+}\right.$and CD45.2 $)$, and NSG (NOD.Cg-Prkdc ${ }^{\text {scid }}$ Ill2rg ${ }^{\text {tm } 1 \mathrm{Wj} /} / \mathrm{SzJ}$; The Jackson Laboratory) were maintained under pathogen-free conditions at the University of Veterinary Medicine Vienna. Genotyping primers and detailed thermocycling conditions are available in the Supplementary Table 5 .

Generation of leukemic cell lines and in vitro deletion of endogenous Cdk8. To generate stable leukemic cell lines, bones of $C d k 8^{f l / f l} M x 1 C r e$ or $C d k 8^{f l / f l} V a v$-Cre were flushed to isolate BM cells for transformation. The phoenix ecotropic $(\varphi \mathrm{NX}$ Eco) packaging system was used to produce supernatant containing pMSCV-IRESGFP-based BCR-ABL1 ${ }^{185+}$ retroviral pseudoparticles. Therefore, phoenix cells were transfected with retroviral vectors using TurboFect ${ }^{\circledR}$ Transfection Reagent (Qiagen). Cells were seeded 1 day before the transfection in six-well plates and grown to $50-70 \%$ confluence. Plasmid DNA $(1.5 \mu \mathrm{g})$ was diluted in $100 \mu \mathrm{L}$ DMEM containing $10 \mathrm{mM}$ HEPES buffer $\mathrm{pH}$ 7.4. $10 \mu \mathrm{L}$ Turbofect ${ }^{\circledR}$ Transfection Reagent was added, vortexed, and incubated $15 \mathrm{~min}$ at room temperature before the transfection mix was added dropwise to the cells. After $24 \mathrm{~h}$, the transfection mix was replaced by RPMI-1640 supplemented with $10 \%$ FCS, $50 \mu \mathrm{M} 2$-mercaptoethanol, and $100 \mathrm{U} / \mathrm{mL}$ penicillin, $100 \mu \mathrm{g} / \mathrm{mL}$ streptomycin (Sigma). Another $24 \mathrm{~h}$ and $48 \mathrm{~h}$ later freshly collected BM cells were infected with the collected supernatant. A010 cells were used for the production of an ecotropic replicationdeficient form of the Abelson virus. To delete $C d k 8$ in the BCR-ABL1 ${ }^{185}+C d k 8^{f l}$ $f_{M}$ xlCre lines, leukemic cells were incubated $24 \mathrm{~h}$ in $1000 \mathrm{U} / \mathrm{mL}$ recombinant IFN$\beta$ (MerckMillipore). Cdk8 deletion was verified by immunoblot analysis.

Colony-formation assay. BM cells from 6-week-old donor mice were transduced with Abelson virus or BCR-ABL1 ${ }^{\text {185+ }}$ supernatants including $7 \mu \mathrm{g} / \mathrm{mL}$ polybrene (Sigma). After $24 \mathrm{~h}$, cells were collected and equal numbers of cells were embedded into growth factor-free methylcellulose (MethoCult $\left.{ }^{\mathrm{m}}\right)$ ). Colonies were counted after 10-14 days.

In vivo leukemia studies. A total of $2500 C d k 8^{f l / f l} \mathrm{BCR}-\mathrm{ABL1} \mathrm{p}^{185+}, C d k 8^{\Delta / \Delta} \mathrm{Vav}-$ Cre BCR-ABL1 $185+$, or $1 \times 10^{5} C d k 8^{f l / f l} \mathrm{v}$-ABL ${ }^{160+}$ or $C d k 8^{\Delta / \Delta}$ Vav-Cre $\mathrm{v}-$ $\mathrm{ABL}^{\mathrm{p} 160+}$ cells were injected via the tail vein into non-irradiated NSG mice. Disease onset was traced by analyzing peripheral blood. Upon appearance of BCR$\mathrm{ABL1}^{\mathrm{p}}{ }^{185+}$ cells in the peripheral blood, we initiated poly(I:C) injections $(200 \mu \mathrm{g})$ to induce Cre recombinase expression and $C d k 8$ deletion, specifically in the transplanted leukemic cells. Poly(I:C) injections were given intraperitoneally every 3 days (four times in total); subsequently, survival of the mice was monitored. BM,
SPL, and blood were collected, cells isolated in phosphate-buffered saline (PBS), and further analyzed by fluorescence-activated cell sorting (FACS).

Homing assay. $C d k 8^{\Delta / \Delta} \mathrm{Vav}$-Cre $L y 5.2^{+}$or $C d k 8^{f l / f l} L y 5.2^{+} \mathrm{BM}$ was mixed with $C D K 8^{+/+} L y 5.1^{+}$BM cells in a 1:1 ratio (containing comparable numbers of LSKs) and injected them i.v. into lethally irradiated (9 Gy) $L y 5.1 / 2^{+}$mice. Ten weeks after transplantation, composition of BM of recipient mice was analysed for repopulation of LSKs and stem cell populations by FACS staining.

For the noncompetitive setting, $1 \times 10^{6}$ BCR-ABL1 ${ }^{\mathrm{p} 185+} \mathrm{Cdk} 8^{f l / f l} L y 5.2^{+}$or BCR-ABL1 $1 \mathrm{p}^{185+} \mathrm{Cdk} 8^{\Delta / \Delta} \mathrm{Vav}$-Cre $L y 5.2^{+}$cells were injected into lethally irradiated (9 Gy) $C d k 8^{+/+} L y 5.1^{+}$mice. After $18 \mathrm{~h}$, mice were euthanized and the BM was analysed for the presence of BCR-ABL1P185+ Ly5.2+ cells by FACS.

Flow cytometry. Single-cell suspensions of the splenocytes, thymus, and BM were prepared. For blood analysis, the erythrocytes were lysed using BD FACS Lysing Solution according to the manufacturer's protocol (BD Bioscience). Hematopoietic stem cell fractions in the BM were identified according to Wilson et al. ${ }^{53}$. Hematopoietic progenitors were characterized by flow cytometry as follows: MCP $\left(\mathrm{Lin}^{-}\right.$,

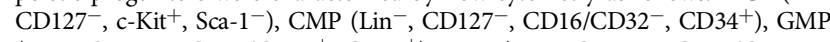
$\left(\mathrm{Lin}^{-}, \mathrm{CD} 127^{-}, \mathrm{CD} 16 / \mathrm{CD} 32^{+}, \mathrm{CD} 34^{+}\right), \mathrm{MEP}\left(\mathrm{Lin}^{-}, \mathrm{CD} 127^{-}, \mathrm{CD} 16 / \mathrm{CD} 32^{-}\right.$, CD34 $4^{-}$), and CLP $\left(\mathrm{Lin}^{-}, \mathrm{CD} 127^{+}, \mathrm{c}-\mathrm{kit}^{\mathrm{mid}}, \mathrm{Sca}-\mathrm{1}^{\mathrm{mid}}\right)$. For cell cycle analysis, cells were stained with propidium iodide (PI) $(50 \mu \mathrm{g} / \mathrm{mL})$ in a hypotonic lysis buffer $(0.1 \%$ sodium citrate, $0.1 \%$ Triton X-100, $100 \mu \mathrm{g} / \mathrm{mL}$ RNAse) and incubated at $37^{\circ} \mathrm{C}$ for 30 min. Analysis of apoptotic fractions was performed by staining with AnnexinV and 7AAD or PI in AnnexinV 10× Staining Buffer (eBiosciences) according to the manufacturer's protocol. Flow cytometry experiments were performed on a $\mathrm{BD}$ FACSCanto II (BD Bioscience) and analyzed using BD FACSDiva V8.0 or FlowJo V10 software. The detailed information to utilized antibodies are available in the Supplementary Table 6.

RNA-seq analysis and GSEA. RNA was isolated from immortalized BCR$\mathrm{ABL}^{\mathrm{p} 185+} C d k 8^{f l / f l}$ and $C d k 8^{\Delta / \Delta} V a v$-Cre cells. Single-end, 50 bp sequencing of libraries prepared with the Lexogen SENSE mRNA-Seq library preparation kit was performed on an Illumina HiSeq-2500 sequencer. After quality control of raw data with FastQC and removement of adapters and low quality reads with Trimmomatic (version 0.36), reads were mapped to the GENECODE M13 genome using STAR (version 2.5.2b) with default parameters. Then, featureCounts from the Subread package (version 1.5.1) was used to obtain gene counts for union gene models. Differentially expressed ( $p$-adjust $<0.05$ and fold change $>2$ ) treatment dependent (Cdk8 $8^{f / f l}$ dimethyl sulfoxide (DMSO) vs. $C d k 8^{f / f l}$ Senexin B; $C d k 8^{\Delta / \Delta} V a v$-Cre DMSO vs. $C d k 8^{\Delta / \Delta}$ Vav-Cre Senexin B) and genotype-dependent (Cdk8 $8^{l / f l}$ vs. $C d k 8^{\Delta / \Delta}$ Vav $\mathrm{Cre}$ ) genes were identified using DESeq2 (version 1.18.1). For the heatmap, centered and scaled regularized log-transformed ${ }^{54}$ library size-normalized counts were visualized using the aheatmap function of the R package NMF (version 0.20.6). The command-line version of GSEA was used for GSEA ${ }^{55}$ and pathway analysis was performed with EnrichR ${ }^{56}$ using either significant up- or downregulated genes (fold

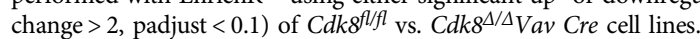

Co-expression matrix. Publicly available datasets of the TARGET pediatric ALL cohort and of 19 TCGA cohorts were obtained from the cBioPortal for Cancer Genomics database ${ }^{57,58}$. RNA-seq RPKM values of CDK8 and various members of the mTOR/PI3K signaling pathways were extracted from these datasets and were used for co-expression analyses. Spearman's correlation was calculated using the cor function of R software (v3.5.1) and the resulting correlation matrix was visualized using the corrplot package. 
Immunoblotting. Whole-cell extracts were lysed in RIPA buffer (50 mM Tris-HCL (pH 7.6), $150 \mathrm{mM} \mathrm{NaCl}, 1 \% \mathrm{NP}-40,0.25 \%$ sodium deoxycholate, $1 \mathrm{mM}$ EDTA; 20 $\mathrm{mM} \beta$-glycero-phosphate, $1 \mathrm{mM}$ sodium vanadate, $1 \mathrm{mM}$ sodium fluoride, $1 \mathrm{mg} / \mathrm{mL}$ aprotinin, $1 \mathrm{mg} / \mathrm{mL}$ leupeptin, and $1 \mathrm{mM}$ phenylmethylsulfonyl fluoride) or in SDSsample buffer. Equal amounts of proteins were separated by SDS polyacrylamide gels and were transferred to nitrocellulose membranes (Whatman ${ }^{\circ}$ Protran ${ }^{\circ}$ ). After

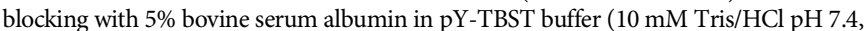
$75 \mathrm{mM} \mathrm{NaCl}, 1 \mathrm{mM}$ EDTA, $0.1 \%$ Tween-20), membranes were probed with primary antibodies (Supplementary Table 7) overnight at $4{ }^{\circ} \mathrm{C}$. Immunoreactive bands were visualized after incubation with the secondary antibody and Clarity Western ECL Substrate (Bio-Rad) using the ChemiDoc ${ }^{\mathrm{TM}}$ Touch (Bio-Rad).

RNA isolation and qRT-PCR. Total RNA was isolated from stable BCR-ABL1 ${ }^{\text {185+ }}$ cell lines. RNA was extracted using the RNeasy Mini Kit (Qiagen). Reverse transcription was performed using the iSCRIPT cDNA synthesis kit following the manufacturer's instructions (Bio-Rad). All quantitative PCRs (qPCRs) were performed in triplicates with Sso Advanced Universal SYBR Green Supermix (Bio-Rad) according to the instructions of the manufacturer. Levels of mRNAs were normalized to hypoxanthine guanine phosphoribosyltransferase (HRPT), $\beta$-actin, or Ube2d2a mRNA. Primer information are available in the Supplementary Table 8.

Inhibitors. For dose-response curves, BCR-ABL1 ${ }^{\mathrm{p} 185+} \mathrm{C} d k 8^{f l / f l}$ or $C d k 8^{\Delta / \Delta}$ Vav-Cre cells were plated in triplicates in 96-well plates. Four thousand cells per well in $100 \mu \mathrm{L}$ of media were incubated $48 \mathrm{~h}$ with inhibitors (Senexin B: Gentaur GmBH/APExBio; MSC2530818, THZ-1, Torin1, and Everolimus: Selleck Chemicals; Cal101, PDK1/ Akt/Flt dual pathway inhibitor, and BAY11-7085: Calbiochem; NVP-2, SEL120-34A: MedChemExpress; Ruxolitinib: Chemietek). Cell viability was assessed using CellTiterGlo (Promega) according to the manufacturer's instructions. Data for each cell line were normalized to the negative control (DMSO, set to $100 \%$ viability) and $50 \%$ inhibitor concentration $\left(\mathrm{IC}_{50}\right.$ ) was determined by using GraphPad Prism ${ }^{\circledR}$ version 5.00. REH, Z119, BV173, BCR-ABL1p185+ $C d k 8^{f l l f l}$, or $C d k 8^{\Delta / \Delta} V a v$-Cre cells were seeded in a six-well dish at a concentration of $10^{6}$ cells $/ \mathrm{mL}$. Senexin B, MSC2530818, or SEL120-34A was added and after $48 \mathrm{~h}$ incubation cells were collected. Cells $\left(10^{6}\right)$ were washed with ice-cold phosphate-buffered saline (PBS) and were boiled $\left(95^{\circ} \mathrm{C}\right)$ for $20 \mathrm{~min}$ in $100 \mu \mathrm{L}$ SDS-sample buffer consisting of 5\% SDS (Biomol), $5 \%$ glycerol (Merck), 2.5\% 2-mercapoethanol, and a trace amount of bromophenol blue sodium salt (Merck) in $375 \mathrm{mM}$ Tris/HCl (pH 6.8). The remaining cells were analyzed by FACS (cell cycle and viability).

Degraders. Synthesis details of YKL-06-101 and JH-XI-10-02 are provided in Supplementary Notes 1 and 2. Proliferation of degrader-exposed human leukemic cell lines and primary ALL patient cells were examined by measuring $3 \mathrm{H}-$ thymidine uptake after $48 \mathrm{~h}$. For immunoblottings and FACS analysis, cells were incubated with the indicated concentrations of the degraders up to $96 \mathrm{~h}$.

Statistical analysis. Kruskal-Wallis test (followed by Dunn's test), one-way analysis of variance (followed by Tukey's multiple comparison test), log-rank (Mantel-Cox) test, Wilcoxon-Mann-Whitney test, and assessment of half maximal inhibitory concentration values were performed using GraphPad Prism ${ }^{\oplus}$ Software version 5.04 and 6.02 . All data are shown as mean \pm SD or otherwise as described in the figure legends. The significance is indicated for each experiment $\left({ }^{*} p<0.05,{ }^{* *} p<0.01,{ }^{* * *} p<0.001,{ }^{* * * *} p<0.0001\right)$.

Study approvals. All animal experiments were approved by the institutional ethics committee and were granted by the national authority (Austrian Federal Ministry of Science and Research) according to Section 8ff of Law for Animal Experiments under license BMWF-68.205/0218-II/3b/2012, and were conducted according to the guidelines of FELASA and ARRIVE.

The ethics committee of the Medical University of Vienna approved the study on human leukemic cells (approval number: 011-2005). All patients gave their written informed consent to participate before BM or blood cells were examined.

Reporting summary. Further information on research design is available in the Nature Research Reporting Summary linked to this article.

\section{Data availability}

The RNA sequencing data have been deposited in the Gene Expression Omnibus (GEO) database under the accession code GSE136923. The source data and uncropped gel pictures underlying Figs. 1a-e, 2a-f, h-j, 3a-i, $4 b-d, f-i, 5 a-b, 6 b-d$, and $7 d-j$, and Supplementary Figs. 1a, 2a-f, 3a-h, 4a-b, 5a-b, $6 \mathrm{~b}-\mathrm{c}$, and $8 \mathrm{a}-\mathrm{d}$ are provided as a source data file. Data that support the findings of this study are available from the authors upon reasonable request.

Received: 12 January 2019; Accepted: 20 September 2019; Published online: 18 October 2019

\section{References}

1. Cao, L. et al. Phylogenetic analysis of CDK and cyclin proteins in premetazoan lineages. BMC Evol. Biol. 14, 10 (2014).

2. Lim, S. \& Kaldis, P. Cdks, cyclins and CKIs: roles beyond cell cycle regulation. Development 140, 3079-3093 (2013).

3. Malumbres, M. et al. Cyclin-dependent kinases. Genome Biol. 15, 122 (2014)

4. Malumbres, M. et al. Mammalian cells cycle without the D-type cyclindependent kinases Cdk4 and Cdk6. Cell 118, 493-504 (2004).

5. Kollmann, K. et al. A kinase-independent function of CDK6 links the cell cycle to tumor angiogenesis. Cancer Cell 24, 167-181 (2013).

6. Kollmann, K. et al. c-JUN promotes BCR-ABL-induced lymphoid leukemia by inhibiting methylation of the 5' region of Cdk6. Blood 117, 4065-4075 (2011).

7. Scheicher, R. et al. CDK6 as a key regulator of hematopoietic and leukemic stem cell activation. Blood 125, 90-101 (2015).

8. Uras, I. Z. et al. Palbociclib treatment of FLT3-ITD+ AML cells uncovers a kinase-dependent transcriptional regulation of FLT3 and PIM1 by CDK6. Blood 127, 2890-2902 (2016).

9. Laderian, B. \& Fojo, T. CDK4/6 inhibition as a therapeutic strategy in breast cancer: palbociclib, ribociclib, and abemaciclib. Semin. Oncol. 44, 395-403 (2017).

10. Thomas, D. et al. Targeting acute myeloid leukemia by dual inhibition of PI3K signaling and $\mathrm{Cdk}^{-}$mediated $\mathrm{Mcl}-1$ transcription. Blood 122, 738-748 (2013).

11. Chipumuro, E. et al. CDK7 inhibition suppresses super-enhancer-linked oncogenic transcription in MYCN-driven cancer. Cell 159, 1126-1139 (2014).

12. Kwiatkowski, N. et al. Targeting transcription regulation in cancer with a covalent CDK7 inhibitor. Nature 511, 616-620 (2014).

13. Arguello, B. F. et al. Flavopiridol induces apoptosis of normal lymphoid cells, causes immunosuppression, and has potent antitumor activity in vivo against human leukemia and lymphoma xenografts. Blood 91, 2482-2490 (2016).

14. Kumar, S. K. et al. Dinaciclib, a novel CDK inhibitor, demonstrates encouraging single-agent activity in patients with relapsed multiple myeloma. Blood 125, 443-448 (2015).

15. Baker, A. et al. The CDK9 inhibitor dinaciclib exerts potent apoptotic and antitumor effects in preclinical models of MLL-rearranged acute myeloid leukemia. Cancer Res. 76, 1158-1169 (2016).

16. Bose, P. et al. Phase I trial of the combination of flavopiridol and imatinib mesylate in patients with Bcr-Abl+ hematological malignancies. Cancer Chemother. Pharmacol. 69, 1657-1667 (2012).

17. Morales, F. \& Giordano, A. Overview of CDK9 as a target in cancer research. Cell Cycle 15, 519-527 (2016).

18. Westerling, T., Kuuluvainen, E. \& Mäkelä, T. P. Cdk8 is essential for preimplantation mouse development. Mol. Cell. Biol. 27, 6177-6182 (2007).

19. McCleland, M. L. et al. Cdk8 deletion in the ApcMin murine tumour model represses EZH2 activity and accelerates tumourigenesis. J. Pathol. 237, 508-519 (2015).

20. Donner, A. J., Szostek, S., Hoover, J. M. \& Espinosa, J. M. CDK8 is a stimulusspecific positive coregulator of p53 target genes. Mol. Cell 27, 121-133 (2007).

21. Galbraith, M. D. et al. HIF1A employs CDK8-mediator to stimulate RNAPII elongation in response to hypoxia. Cell 153, 1327-1339 (2013).

22. Rzymski, T. et al. SEL120-34A is a novel CDK8 inhibitor active in AML cells with high levels of serine phosphorylation of STAT1 and STAT5 transactivation domains. Oncotarget 8, 33779-33795 (2017).

23. Putz, E. M. et al. CDK8-mediated STAT1-S727 phosphorylation restrains NK cell cytotoxicity and tumor surveillance. Cell Rep. 4, 437-444 (2013).

24. Bancerek, J. et al. CDK8 kinase phosphorylates transcription factor STAT1 to selectively regulate the interferon response. Immunity 38, 250-262 (2013).

25. Firestein, R. et al. CDK8 is a colorectal cancer oncogene that regulates $\beta$ catenin activity. Nature 455, 547-551 (2008).

26. Firestein, R., Shima, K., Nosho, K., Irahara, N. \& Baba, Y. CDK8 expression in 470 colorectal cancers in relation to $\beta$-catenin activation, other molecular alterations and patient survival. Int J. Cancer 126, 2863-2873 (2011).

27. Kapoor, A. et al. The histone variant macroH2A suppresses melanoma progression through regulation of CDK8. Nature 468, 1105-1109 (2010).

28. Nakamura, A., Nakata, D., Kakoi, Y. \& Kunitomo, M. CDK8/19 inhibition induces premature G1 / S transition and ATR-dependent cell death in prostate cancer cells. Oncotarget 9, 13474-13487 (2018).

29. McDermott, M. S. J. et al. Inhibition of CDK8 mediator kinase suppresses estrogen dependent transcription and the growth of estrogen receptor positive breast cancer. Oncotarget 8, 12558-12575 (2017).

30. $\mathrm{Gu}, \mathrm{W}$. et al. Tumor-suppressive effects of CDK8 in endometrial cancer cells. Cell Cycle 12, 987-999 (2013).

31. Pelish, H. E. et al. Mediator kinase inhibition further activates superenhancer-associated genes in AML. Nature 526, 273-276 (2015).

32. Piccaluga, P. P., Paolini, S. \& Martinelli, G. Tyrosine kinase inhibitors for the treatment of Philadelphia chromosome-positive adult acute lymphoblastic leukemia. Cancer 110, 1178-1186 (2007). 
33. Yang, K. \& Fu, L. W. Mechanisms of resistance to BCR-ABL TKIs and the therapeutic strategies: a review. Crit. Rev. Oncol. Hematol. 93, 27-292 (2015).

34. Hardy, R. R. et al. Immunological reviews B-cell commitment, development and selection. Immunol. Rev. 175, 23-32 (2000).

35. Hardy, R. R. B-cell commitment: deciding on the players. Curr. Opin. Immunol. 15, 158-165 (2003).

36. Winter, G. E. et al. Phthalimide conjugation as a strategy for in vivo target protein degradation. Science 348, 1376-1381 (2015).

37. Hatcher, J. M. et al. Development of highly potent and selective steroidal inhibitors and degraders of CDK8. ACS Med. Chem. Lett. 9, 540-545 (2018).

38. Thoreen, C. C. et al. An ATP-competitive mammalian target of rapamycin inhibitor reveals rapamycin-resistant functions of mTORC1. J. Biol. Chem. 284, 8023-8032 (2009).

39. Philip, S., Kumarasiri, M., Teo, T., Yu, M. \& Wang, S. Cyclin-dependent kinase 8: a new hope in targeted cancer therapy? J. Med. Chem. 61, 5073-5092 (2017).

40. Clarke, P. A. et al. Assessing the mechanism and therapeutic potential of modulators of the human mediator complex-associated protein kinases. Elife 5, e20722 (2016)

41. Aranda-Orgilles, B. et al. MED12 regulates HSC-specific enhancers independently of mediator kinase activity to control hematopoiesis. Cell Stem Cell 19, 784-799 (2016).

42. Berger, A. et al. PAK-dependent STAT5 serine phosphorylation is required for BCR-ABL-induced leukemogenesis. Leukemia 28, 629-641 (2014).

43. Witalisz-Siepracka, A. et al. NK cell-specific CDK8 deletion enhances antitumor responses. Cancer Immunol. Res. 6, 458-466 (2018).

44. Koehler, M. F. T. et al. Development of a potent, specific CDK8 kinase inhibitor which phenocopies CDK8/19 knockout cells. ACS Med. Chem. Lett. 7, 223-228 (2016).

45. Carayol, N. et al. Critical roles for mTORC2- and rapamycin-insensitive mTORC1-complexes in growth and survival of BCR-ABL-expressing leukemic cells. Proc. Natl Acad. Sci. USA 107, 12469-12474 (2010).

46. Feng, D. et al. mTORC1 down-regulates cyclin-dependent kinase 8 (CDK8) and cyclin C (CycC). PLoS ONE 10, e0126240 (2015).

47. Marti, R. M., Wasik, M. A. \& Kadin, M. E. Constitutive secretion of GM-CSF by three different cell lines derived from a single patient with a progressive cutaneous lymphoproliferative disorder. Cytokine 8, 323-329 (1996).

48. Morikawa, S., Tatsumi, E., Baba, M., Harada, T. \& Yasuhira, K. Two E-rosetteforming lymphoid cell lines. Int. J. Cancer 21, 166-170 (1978).

49. Fellmann, C. et al. An optimized microRNA backbone for effective singlecopy RNAi. Cell Rep. 5, 1704-1713 (2013).

50. Zuber, J. et al. RNAi screen identifies Brd4 as a therapeutic target in acute myeloid leukaemia. Nature 478, 524-528 (2011).

51. Kühn, R., Schwenk, F., Aguet, M. \& Rajewsky, K. Inducible gene targeting in mice. Science 269, 1427-1429 (1995)

52. Georgiades, P. et al. vavCre transgenic mice: a tool for mutagenesis in hematopoietic and endothelial lineages. Genesis 34, 251-256 (2002).

53. Wilson, A., Laurenti, E. \& Trumpp, A. Balancing dormant and self-renewing hematopoietic stem cells. Curr. Opin. Genet. Dev. 19, 461-468 (2009).

54. Love, M. I., Huber, W. \& Anders, S. Moderated estimation of fold change and dispersion for RNA-seq data with DESeq2. Genome Biol. 15, 550 (2014).

55. Subramanian, A. et al. Gene set enrichment analysis: a knowledge-based approach for interpreting genome-wide expression profiles. Proc. Natl Acad. Sci. USA 102, 15545-15550 (2005).

56. Kuleshov, M. V. et al. Enrichr: a comprehensive gene set enrichment analysis web server 2016 update. Nucleic Acids Res. 44, W90-W97 (2016)

57. Gao, J. et al. Integrative analysis of complex cancer genomics and clinical profiles using the cBioPortal. Sci. Signal. 6, pl1 (2013).

58. Cerami, E. et al. The cBio cancer genomics portal: an open platform for exploring multidimensional cancer genomics data. Cancer Discov. 2, 401-404 (2012).

\section{Acknowledgements}

We thank the European Conditional Mouse Mutagenesis Consortium and Mohammed Selloum (Yann Herault Group) for providing the Cdk8 $8^{\operatorname{tm} l c(E U C O M M) H m g u}$ mouse strain. We are grateful to Thomas Ruelicke and Thomas Kolbe for their help in acquiring the mice and to all members of the mouse facility. Z119 cells were kindly provided to J. V. Melo by Zeev Estrov. For technical support, we want to thank Sabine Fajmann and Philipp Jodl. We are deeply indebted to Graham Tebb for critical discussions and editing of the manuscript. This work was supported by grants from Austrian Science Foundation (FWF) SFB 4701-B20 to P.V., F4704-B20 to P.V., F4706-B20 to V.S., P28571-B21 to V.S. P27248-B28 to D.F., and SFB6107 to V.S.

\section{Author contributions}

V.S. was the principal investigator and takes primary responsibility for the article. V.S and N.S.G. designed and supervised the study. I.M., A.B.-B., M.P.-M., L.E., V.M.K., I.Z.U., E.G., K.B., and A.S. performed and analyzed experiments. T.Z., Y.L., J.M.H., and Y.L. synthesized and characterized the degraders. R.G. and G.H. performed bioinformatics analysis. P.V. provided patient materials and human leukemic cell lines. J.V.M. provided the Z199 cell line. M.R., S.K., F.G., J.Z., D.F., A.H.K., N.P.K., and T.W. contributed to interpretation of the data and revised the manuscript with regard to critical intellectual content. I.M. and V.S. wrote the manuscript.

\section{Competing interests}

N.S.G. is a founder, science advisory board member (SAB), and equity holder in Gatekeeper, Syros, Petra, C4, B2S, and Soltego. The Gray lab receives or has received research funding from Novartis, Takeda, Astellas, Taiho, Janssen, Kinogen, Voronoi, Her2llc, Deerfield, and Sanofi. N.P.K., N.S.G., T.Z., and Y.L. are inventors on the patent WO2017/ 185034 that covers degrader YKL-06-101. N.S.G. and J.M.H. are inventors on a patent that covers JH-XI-10-02. The remaining authors declare no competing interests.

\section{Additional information}

Supplementary information is available for this paper at https://doi.org/10.1038/s41467019-12656-x.

Correspondence and requests for materials should be addressed to V.S.

Peer review information Nature Communications thanks Jonathan Schatz and the other, anonymous, reviewer(s) for their contribution to the peer review of this work.

Reprints and permission information is available at http://www.nature.com/reprints

Publisher's note Springer Nature remains neutral with regard to jurisdictional claims in published maps and institutional affiliations.

Open Access This article is licensed under a Creative Commons Attribution 4.0 International License, which permits use, sharing, adaptation, distribution and reproduction in any medium or format, as long as you give appropriate credit to the original author(s) and the source, provide a link to the Creative Commons license, and indicate if changes were made. The images or other third party material in this article are included in the article's Creative Commons license, unless indicated otherwise in a credit line to the material. If material is not included in the article's Creative Commons license and your intended use is not permitted by statutory regulation or exceeds the permitted use, you will need to obtain permission directly from the copyright holder. To view a copy of this license, visit http://creativecommons.org/ licenses/by/4.0/.

(C) The Author(s) 2019 